Pacific Journal of Mathematics

ALGEBRAS OF UNBOUNDED SCALAR-TYPE SPECTRAL

Peter Gerard Dodos and Bernardus de Patter 


\title{
ALGEBRAS OF UNBOUNDED SCALAR-TYPE SPECTRAL OPERATORS
}

\author{
P. G. Dodds AND B. DE PAgter
}

If $P: \Sigma \rightarrow \mathscr{L}(X)$ is a closed spectral measure in the quasicomplete locally convex space $X$ and if $T$ is a densely defined linear operator in $X$ with domain invariant under each operator of the form $\int_{\Omega} f d P$, with $f$ a complex bounded $\Sigma$-measurable function then $T$ is closable and there exists a complex $\Sigma$-measurable function $f$ such that the closure of $T$ is the spectral integral $\int_{\Omega} f d P$ if and only if $T$ leaves invariant each closed subspace of $X$ which is invariant under the range of the spectral measure $P$.

0. Introduction. Let $X$ be a complex locally convex Hausdorff space, assumed quasicomplete throughout. Let $\mathscr{L}(X)$ be the space of all continuous linear operators in $X$ equipped with the topology of pointwise convergence in $X$. Let $P: \Sigma \rightarrow \mathscr{L}(X)$ be a spectral measure with domain $\Sigma$ a $\sigma$-algebra of subsets of some point set $\Omega$ and with range a closed subset of $\mathscr{L}(X)$. The intention of the present paper is to characterize those densely defined operators $T$ on $X$ that can be represented as a spectral integral $\int_{\Omega} f d P$ for some complex, $\Sigma$-measurable function $f$ on $\Omega$. More precisely, we show (Theorem 6.2) that if $T$ is densely defined with domain $\mathscr{D}(T)$ invariant under each operator of the form $\int_{\Omega} f d P$ with $f$ a bounded, complex $\Sigma$-measurable function, then $T$ is closable and there exists a complex $\Sigma$-measurable function $f$ on $\Omega$ such that $T$ is given by the spectral integral $\int_{\Omega} f d P$ if and only if $T$ leaves invariant each closed linear subspace of $\mathscr{L}(X)$ which is invariant under the range of the spectral measure $P$. That part of the conclusion which asserts that $T$ is closable appears to be new, even if $X$ is a Banach space, and is not valid if the stated assumption on $\mathscr{D}(T)$ is replaced by the weaker assumption that $\mathscr{D}(T)$ is invariant under merely the range of $P$. Under this weaker assumption on $\mathscr{D}(T)$, the above characterization remains valid if, in addition, $T$ is assumed closed (Corollary 6.3) and this result extends to the locally convex setting a characterization of scalar-type spectral operators given by Sourour [22] for the case that $X$ is Banach. Further, for everywhere defined operators on locally convex space, our results specialize to the reflexivity criteria of [6], [4]. 
The method of the present paper is based on the observation implicit in [4] and explicit in [5] (Proposition 2.2) that the strongly closed algebra generated by the range of $P$ admits an order structure with particularly strong algebraic and topological properties and our approach is to exploit the very special features of this order structure by appropriately refining the techniques of [4], thus bypassing those Banach space methods based on the existence of a (so-called) "Bade functional", which are not valid in the locally convex setting, even for metrizable spaces. More precisely, if $\mathscr{M}$ denotes the range of $P$, and $\langle\mathscr{M}\rangle$ the strongly closed algebra in $\mathscr{L}(X)$ generated by the range of $P$, we show first that the set of all densely defined linear mappings in $X$ which admit a representation as a spectral integral $\int_{\Omega} f d P$ for some complex, $\Sigma$-measurable function $f$, has the structure of a Dedekind complete $f$-algebra $\langle\mathscr{M}\rangle_{\infty}$ which may be identified with the universal completion of the $f$-algebra $\langle\mathscr{M}\rangle$. It is then shown that the restriction of $\langle\mathscr{M}\rangle_{\infty}$ to each cyclic subspace $\mathscr{M}[x], x \in X$, itself a Dedekind complete Riesz space for the natural ordering induced by that of $\langle\mathscr{M}\rangle$, coincides precisely with the Riesz space $\operatorname{Orth}^{\infty}(\mathscr{M}[x])$ of all linear, densely defined, order bounded linear maps in $\mathscr{M}[x]$ which are band preserving. If now $T$ has domain satisfying the condition stated in the first paragraph and leaves invariant each $\mathscr{M}$-invariant subspace in $X$ then the restriction of $T$ to each cyclic subspace induces a densely defined linear mapping which is band-preserving. A key point in our argument is then to use appropriate extensions of the result of [14] to show that $T$ is automatically order bounded and thus given in a local sense by (the restriction of) an element of $\langle\mathscr{M}\rangle_{\infty}$.

With a view to applications of the main results we consider certain aspects of the spectral theory of (unbounded) scalar-type spectral operators. In particular, we show in $\$ 6$ below, that each scalar-type spectral operator $T$ (in the sense of Dunford) admits a uniquely determined resolution of the identity which commutes with each continuous operator commuting with $T$ and whose support coincides with the spectrum of $T$. Special cases of these results are of course well known and contained in [12] and [17] for everywhere defined operators and in [20] for a restricted class of densely defined operators. Rather than reduce our results to those known for the continuous case, we have preferred here to give a treatment which applies simultaneously to both continuous and unbounded scalartype operators, basing our approach as closely as possible on that outlined in [7] Chapter XV, but using the established algebraic and order structure as a tool for computation, the link between the present and earlier approaches being supplied by the abstract spectral theorem of Freudenthal. Finally, we show that the main results of the paper provide the 
tools necessary to extend to the locally convex setting, certain reflexivity theorems for (in general non-commutative) closed algebras of operators on Banach spaces containing Boolean algebras of uniform multiplicity one, due to Sourour [21] and Rosenthal and Sourour [19].

The authors wish to thank A. R. Sourour for bringing to their attention the papers [21], [22], [18], [19], [13]. Part of this paper was written while the authors were guests of the Institut für Mathematik, Johannes Kepler Universität Linz and the authors wish to thank J. B. Cooper and members of the Institut for the kind hospitality extended during the period of their visit.

1. Some preliminary information. This paper is based mainly on the techniques developed in [4] (and [5]). We assume that the reader has some familiarity with the theory of Riesz spaces. For terminology and basic facts used we refer to the books [11], [24] (for some information on topological Riesz spaces see [1]). The purpose of the present section is to gather for the convenience of the reader some of the results obtained in [4] (and [5]) concerning the structure of strongly closed operator algebras generated by Boolean algebras of projections, and the corresponding cyclic subspaces.

Let $X$ be a (complex) locally convex vector space. We assume that $X$ is quasi-complete. By $\mathscr{L}^{\#}(X)$ we denote the space of all linear operators in $X$, and by $\mathscr{L}(X)$ we denote the subspace of $\mathscr{L}^{\#}(X)$ consisting of all continuous operators. In $\mathscr{L}^{\#}(X)$ and $\mathscr{L}(X)$ we consider the strong operator topology (i.e., topology of pointwise convergence). Note that $\mathscr{L}^{\#}(X)$ is quasi-complete. Let $\mathscr{M}$ be an equicontinuous Boolean algebra of projections in $X$, i.e., $\mathscr{M}$ is an equicontinuous collection of mutually commuting idempotents in $\mathscr{L}(X)$, partially ordered by range inclusion, which is a Boolean algebra with respect to the lattice operations defined by $E \wedge F=E F$ and $E \vee F=E+F-E F$ for $E, F \in \mathscr{M}$. We always assume that $I \in \mathscr{M}$. We denote by $\langle\mathscr{M}\rangle$ the strongly closed subalgebra of $\mathscr{L}(X)$ generated by $\mathscr{M}$, i.e., $\langle\mathscr{M}\rangle$ is the closure of $M=\left\{\sum_{l=1}^{n} \alpha_{i} E_{l}\right.$ : $\left.E_{i} \in \mathscr{M}, \alpha_{i} \in \mathbf{C}(i=1, \ldots, n), n \in \mathbf{N}\right\}$. Moreover, $\langle\mathscr{M}\rangle^{\#}$ denotes the closure of $M$ in $\mathscr{L}^{\#}(X)$. We recall that $\mathscr{M}$ is called strongly equicontinuous if $E_{n} \rightarrow 0$ (strongly) for any disjoint sequence $\left\{E_{n}\right\}_{n=1}^{\infty}$ in $\mathscr{M}$. It should be observed that the range of a ( $\sigma$-additive) equicontinuous spectral measure is a strongly equicontinuous Boolean algebra of projections. Furthermore, $\mathscr{M}$ is called Bade-complete if $\mathscr{M}$ is complete as a Boolean algebra and $E_{\alpha} \uparrow E$ in $\mathscr{M}$ implies that $E_{\alpha} \rightarrow E$ with respect to the strong operator topology. As observed in [4], Proposition 4.7, an equicontinuous Boolean algebra $\mathscr{M}$ is strongly equicontinuous if and only 
if the strong closure $\overline{\mathscr{M}}$ is Bade-complete. Therefore, for the study of $\langle\mathscr{M}\rangle$ and $\langle\mathscr{M}\rangle^{\#}$ we may assume, without loss of generality, that $\mathscr{M}$ is Bade-complete. From now on we assume that $\mathscr{M}$ is an equicontinuous Bade-complete Boolean algebra of projection in $X$.

An element $T \in M$ has a unique standard representation $T=$ $\sum_{i=1}^{n} \lambda_{i} E_{i}$, where $E_{i} \neq 0$ in $\mathscr{M}$ are such that $E_{i} E_{j}=0(i \neq j), \sum_{i=1}^{n} E_{i}=I$ and $\lambda_{1}, \ldots, \lambda_{n}$ are mutually different scalars. Now it is clear that $M$ has the structure of a complex Riesz space, where the absolute value of $T=\sum_{i=1}^{n} \lambda_{i} E_{i}$ (standard representation) is given by $|T|=\sum_{i=1}^{n}\left|\lambda_{i}\right| E_{i}$. Furthermore, with respect to the topology induced by $\mathscr{L}(X), M$ is a complex locally solid Riesz space ([4], Lemma 3.2). Since the strong closure $\langle\mathscr{M}\rangle^{\#}$ of $M$ in $\mathscr{L}^{\#}(X)$ is, in fact, the topological completion of $M$ ([4], Proposition 3.12) the lattice operations extend to $\langle\mathscr{M}\rangle^{\#}$. Now $\langle\mathscr{M}\rangle^{\#}$ has the structure of a complex Dedekind complete Riesz space with locally convex locally solid Lebesgue topology ([4], Proposition 3.6). In particular, $0 \leq T_{\alpha} \uparrow T$ in $\langle\mathscr{M}\rangle^{\#}$ implies that $T_{\alpha} \rightarrow T$ strongly. Moreover, as shown in [4], Proposition 4.2, with respect to composition as multiplication, $\langle\mathscr{M}\rangle^{\#}$ is a complex $f$-algebra (i.e., the product of positive elements is likewise positive and if $S, T, R \in\langle\mathscr{M}\rangle^{\#}$ with $S \perp T$, then $R S \perp T$ and $S R \perp T$; see [24], Chapter 20 for general properties of $f$-algebras). In particular, $\langle\mathscr{M}\rangle^{\#}$ is commutative. As observed at the end of $\S 2$ in [5], $\langle\mathscr{M}\rangle$ is an order ideal in $\langle\mathscr{M}\rangle^{\#}$, and therefore $\langle\mathscr{M}\rangle$ has the structure of a Dedekind complete complex $f$-algebra with locally solid Lebesgue topology (but $\langle\mathscr{M}\rangle$ is in general not topologically complete; however, if $\mathscr{L}(X)$ is sequentially complete, then $\langle\mathscr{M}\rangle^{\#}=\langle\mathscr{M}\rangle$, as shown in [4], Proposition 4.3). We note that, since we assume that $\mathscr{M}$ is Bade-complete, the Boolean algebra of idempotents in $\langle\mathscr{M}\rangle$ is precisely $\mathscr{M}$ ([4], Proposition 4.4).

Next we say a few words about the structure of the cyclic subspaces of $\mathscr{M}$. Fix $x \in X$ and put $M(x)=\{T x: T \in \mathscr{M}\}$. The mapping $x \rightarrow T x$ from $M$ onto $M(x)$ induces the structure of a complex Riesz space in $M(x)$, and the topology induced by $X$ in $M(x)$ is locally solid ([4], Lemma 3.3). Let $\mathscr{M}(x)$ be the closure of $M(x)$ in $X$. Since $\mathscr{M}(x)$ is equal to the topological completion of $M(x), \mathscr{M}(x)$ has the structure of a complex Dedekind complete Riesz space with a complete locally solid convex Lebesgue topology ([4], Proposition 3.9).

Clearly, $\mathscr{M}(x)$ is $T$-invariant for any $T \in\langle\mathscr{M}\rangle^{\#}$. The restriction $T[x]$ of $T$ to $\mathscr{M}(x)$ is, in fact, an orthomorphism in the Riesz space $\mathscr{M}(x)$ (i.e., $T[x]$ is order bounded and $y \perp z$ in $\mathscr{M}(x)$ implies $T[x] y \perp z$; see [24], Chapter 20 for the general theory of orthomorphisms). The 
mapping $T \rightarrow T[x]$ from $\langle\mathscr{M}\rangle^{\#}$ into the space $\operatorname{Orth}(\mathscr{M}(x))$ of all orthomorphisms in $\mathscr{M}(x)$ is a Riesz and algebra homomorphism ([4], Propositions 4.1 and 4.2). This homomorphism is, in general, not surjective. However, denoting by $\langle\mathscr{M}\rangle_{I}$ the principal order ideal in $\langle\mathscr{M}\rangle$ generated by the identity, and, as usual, denoting by $Z(\mathscr{M}(x))$ the centre of $\mathscr{M}(x)$ (i.e., $Z(\mathscr{M}(x))$ is the principal order ideal generated by the identity in $\operatorname{Orth}(\mathscr{M}(x)))$, the homomorphism maps $\langle\mathscr{M}\rangle_{I}$ onto $Z(\mathscr{M}(x))$ ([4], Proposition 4.10). In particular, the band projections in $\mathscr{M}(x)$ are precisely the operators of the form $E[x]$ with $E \in \mathscr{M}$.

2. Scalar-type spectral operators. Throughout this section, $X$ will denote a quasi-complete locally convex space, and $\mathscr{L}(X)$ will denote the space of all continuous linear operators in $X$. In $\mathscr{L}(X)$ we consider the strong topology. In this section we will be concerned with linear operators $T: \mathscr{D}(T) \rightarrow X$, where the domain $\mathscr{D}(T)$ is a linear subspace of $X$. If $T_{1}$ and $T_{2}$ are such operators, then we write $T_{1} \subseteq T_{2}$ if $\mathscr{D}\left(T_{1}\right) \subseteq \mathscr{D}\left(T_{2}\right)$ and $T_{1} x=T_{2} x$ for all $x \in \mathscr{D}\left(T_{1}\right)$. Suppose now that $\mathscr{M}$ is a Bade complete Boolean algebra of projections in $X$ and let $\langle\mathscr{M}\rangle$ be the strongly closed subalgebra of $\mathscr{L}(X)$ generated by $\mathscr{M}$.

Definition 2.1. The linear operator $T$ will be called a spectral element with respect to $\mathscr{M}$ if there exists a sequence $\left\{E_{n}\right\}_{n=1}^{\infty}$ in $\mathscr{M}$ such that

(i) $E_{n} \uparrow I$ in $\mathscr{M}$;

(ii) $E_{n}(X) \subseteq \mathscr{D}(T)$ and $T E_{n} \in\langle\mathscr{M}\rangle$ for all $n$;

(iii) $T x=\lim _{n \rightarrow \infty} T E_{n} x$ for all $x \in \mathscr{D}(T)$.

Such a sequence $\left\{E_{n}\right\}$ will be called determining for $T$.

It is clear from Definition 2.1 above that each spectral element $T$ is densely defined. Further, it is easily checked that if $T$ is a spectral element with respect to $\mathscr{M}$ and if $\left\{F_{n}\right\} \subseteq \mathscr{M}$ is any sequence for which $F_{n} \uparrow_{n} I$ holds in $\mathscr{M}$, and such that $F_{n}(X) \subseteq \mathscr{D}(T)$ and $T F_{n} \in\langle\mathscr{M}\rangle$ for all $n \in \mathbf{N}$, then also $\left\{F_{n}\right\}$ is a determining sequence for $T$. In particular if $\left\{E_{n}\right\} \subseteq \mathscr{M}$ is a determining sequence for $T$, and if $\left\{F_{n}\right\} \subseteq \mathscr{M}$ is a sequence such that $F_{n} \leq E_{n}$ for all $n \in \mathrm{N}$ and $F_{n} \uparrow_{n} I$ holds in $\mathscr{M}$, then $\left\{F_{n}\right\}$ is a determining sequence for $T$. It follows that if $S$ and $T$ are spectral elements with respect to $\mathscr{M}$ then there exists a sequence $\left\{E_{n}\right\} \subseteq \mathscr{M}$ which is determining for $S$ and $T$ simultaneously.

Spectral elements $T, T^{\prime}$ are called equivalent, written $T \sim T^{\prime}$ if there exists a sequence $\left\{E_{n}\right\} \subseteq \mathscr{M}$ with $E_{n} \uparrow_{n} I$ such that $E_{n}(X) \subseteq \mathscr{D}(T) \cap$ $\mathscr{D}\left(T^{\prime}\right)$ and $T E_{n}=T^{\prime} E_{n} \in\langle\mathscr{M}\rangle$ for all $n \in \mathbf{N}$. It is clear that such a 
sequence $\left\{E_{n}\right\}$ is determining for both $T$ and $T^{\prime}$. Further, it is clear that the relation so defined is reflexive and symmetric. Since $E_{n} F_{n} \uparrow I$ whenever $\left\{E_{n}\right\}$ and $\left\{F_{n}\right\}$ are sequences in $\mathscr{M}$ with $E_{n} \uparrow I$ and $F_{n} \uparrow I$, it follows readily that the given relation is transitive as well, and so is an equivalence relation. If $T$ is a spectral element, we denote by [T] the equivalence class of spectral elements determined by $T$. It is not difficult to see that spectral elements $T$ and $T^{\prime}$ are equivalent if and only if there exists a spectral element $S$ such that $S \subseteq T$ and $S \subseteq T^{\prime}$.

The following Proposition plays a basic role in what follows, and should be compared with XVIII 2.6 of [7].

Proposition 2.2. If $T$ is a spectral element with respect to $\mathscr{M}$ then there exists a unique maximal representative $\dot{T} \in[T]$, which has the following properties:

(i) $\dot{T}$ is densely defined and closed.

(ii) $\mathscr{D}(\dot{T})$ is $\mathscr{M}$-invariant and $\dot{T} E x=E \dot{T} x$ for all $E \in \mathscr{M}$ and $x \in$ $\mathscr{D}(\dot{T})$. Moreover, if $T_{0}$ is any representative of $[T]$ and if $\left\{F_{n}\right\} \subseteq \mathscr{M}$ is any determining sequence for $T_{0}$, then

(iii) $F_{n}(X) \subseteq \mathscr{D}(\dot{T})$ and $\dot{T} F_{n}=T_{0} F_{n}$ for all $n$.

(iv) $x \in \mathscr{D}(\dot{T})$ if and only if $\lim _{n \rightarrow \infty} T_{0} F_{n} x$ exists, in which case $\dot{T} x=\lim _{n \rightarrow \infty} T_{0} F_{n} x$.

Proof. Let $\left\{E_{n}\right\}$ be a determining sequence for the given spectral element $T$. Define $\mathscr{D}(\dot{T})=\left\{x \in X: \lim _{n \rightarrow \infty} T E_{n} x\right.$ exists $\}$ and if $x \in$ $\mathscr{D}(\dot{T})$ define $\dot{T} x=\lim _{n \rightarrow \infty} T E_{n} x$. It is clear that $\dot{T}$ is a spectral element with determining sequence $\left\{E_{n}\right\}$, and that $T \subseteq \dot{T}$, so $\dot{T} \in[T]$. Note that $\dot{T} E_{n}=T E_{n}$ for all $n$. Furthermore, observe that $\dot{T} E_{n} z=E_{n} \dot{T} z$ for all $z \in \mathscr{D}(\dot{T})$ and all $n$. In fact, $z \in \mathscr{D}(\dot{T})$ implies that

$$
E_{n} T z=\lim _{m \rightarrow \infty} E_{n}\left(T E_{m} z\right)=\lim _{m \rightarrow \infty}\left(T E_{m} E_{n}\right) z=T E_{n} z=\dot{T} E_{n} z .
$$

We now show that $\dot{T}$ is closed. Suppose that $\left\{x_{\tau}\right\} \subseteq \mathscr{D}(\dot{T}), x, y \in X$ are such that $x_{\tau} \rightarrow x$ and $\dot{T} x_{\tau} \rightarrow y$. For any $E_{n}$ we have $T E_{n} \in\langle\mathscr{M}\rangle$, so

$$
T E_{n} x=\lim _{\tau} T E_{n} x_{\tau}=\lim _{\tau} E_{n} \dot{T} x_{\tau}=E_{n}\left(\lim _{\tau} \dot{T} x_{\tau}\right)=E_{n} y .
$$

Hence, since $E_{n} \uparrow I$, we find that

$$
\lim _{n \rightarrow \infty} T E_{n} x=\lim _{n \rightarrow \infty} E_{n} y=y,
$$

which implies that $x \in \mathscr{D}(\dot{T})$ and $\dot{T} x=y$.

To see that $\mathscr{D}(\dot{T})$ is $\mathscr{M}$-invariant, let $E \in \mathscr{M}$ and suppose that $x \in \mathscr{D}(\dot{T})$. Then $T E_{n}(E x)=E\left(T E_{n} x\right)$ for all $n$, and so $\lim _{n \rightarrow \infty} T E_{n}(E x)$ $=E \dot{T} x$, which shows that $E x \in \mathscr{D}(\dot{T})$ and $\dot{T} E x=E \dot{T} x$. 
Next we prove that $\dot{T}$ is maximal in [T]. To this end, take any $S \in[T]$, and we will show that $S \subseteq \dot{T}$. Since $S$ and $T$ are equivalent, there exists a sequence $\left\{F_{n}\right\} \subseteq \mathscr{M}$ such that $F_{n} \uparrow I, F_{n}(X) \subseteq \mathscr{D}(S) \cap \mathscr{D}(T)$ and $T F_{n}=S F_{n} \in\langle\mathscr{M}\rangle$ for all $n$. As observed earlier, $\left\{F_{n}\right\}$ is a determining sequence for both $S$ and $T$. Replacing $F_{n}$ by $F_{n} E_{n}$, we may assume that $F_{n} \leq E_{n}$ for all $n$. For $x \in X$ we then get

$$
F_{m}\left(T E_{n} x\right)=\left(T E_{n}\right) F_{m} x=\left(T F_{m}\right) E_{n} x=\left(S F_{m}\right) E_{n} x=E_{n}\left(S F_{m} x\right)
$$

for all $m, n=1,2, \ldots$ Hence, if $x \in \mathscr{D}(S)$, then

$$
T E_{n} x=\lim _{m \rightarrow \infty} F_{m}\left(T E_{n} x\right)=\lim _{m \rightarrow \infty} E_{n}\left(S F_{m} x\right)=E_{n}\left(\lim _{m \rightarrow \infty} S F_{m} x\right)=E_{n} S x
$$

and so $\lim _{n \rightarrow \infty} T E_{n} x=\lim _{n \rightarrow \infty} E_{n} S x=S x$. Therefore, $x \in \mathscr{D}(\dot{T})$ and $\dot{T} x=S x$ for all $x \in \mathscr{D}(S)$, i.e., $S \subseteq \dot{T}$. Thus, $\dot{T}$ is the largest element in $[T]$ and is therefore unique. Since we can construct this maximal element starting with any representative of $[T]$ and any determining sequence, (iii) and (iv) of the Proposition follow at once, and the proof is complete.

It is now appropriate to make the following definition; see, however, the discussion in $§ 6$ below.

Definition 2.3. A spectral element $T$ with respect to $\mathscr{M}$ will be called a spectral operator of scalar-type with respect to $\mathscr{M}$ if there exists a determining sequence $\left\{E_{n}\right\}$ for $T$ such that $\mathscr{D}(T)=\{x \in X$ : $\lim _{n \rightarrow \infty} T E_{n} x$ exists $\}$ and $T x=\lim _{n \rightarrow \infty} T E_{n} x$ for all $x \in \mathscr{D}(T)$.

It is an immediate consequence of (iii) and (iv) of Proposition 2.2 that if $T$ is a scalar-type spectral operator, then $T$ is precisely the unique maximal representive $\dot{T}$ of $[T]$. Moreover, if $T$ is any spectral element, then the unique maximal representative $\dot{T}$ of $[T]$ is a scalar-type spectral operator. We have the following immediate consequence of parts (i), (ii) of Proposition 2.2.

COROLLARY 2.4. If $T$ is a scalar-type spectral operator (with respect to $\mathscr{M})$, then

(i) $T$ is densely defined and closed

(ii) $\mathscr{D}(T)$ is $\mathscr{M}$-invariant and $T E x=E T x$ for all $x \in \mathscr{D}(T)$ and $E \in \mathscr{M}$

(iii) If $Y \subseteq X$ is a closed M-invariant subspace of $X$, then $T(\mathscr{D}(T) \cap Y) \subseteq Y$.

Let $\langle\mathscr{M}\rangle^{\#}$ denote the strongly closed algebra generated by $\mathscr{M}$ in $\mathscr{L}^{\#}(X)$, the space of all (everywhere defined) linear operators on $X$. Take $T \in\langle\mathscr{M}\rangle^{\#}$, then there exists a sequence $\left\{E_{n}\right\}_{n=1}^{\infty}$ in $\mathscr{M}$ such that $E_{n} \uparrow I$ 
in $\mathscr{M}$ and $\left|T E_{n}\right| \leq n I$ for all $n$ (we use here the canonical Riesz space structure of $\left.\langle\mathscr{M}\rangle^{\#}\right)$. In fact, let $E_{n}$ be the component of the identity operator $I$ in the band generated by the operator $(n I-|T|)^{+}$in $\langle\mathscr{M}\rangle^{\#}$. Hence, the sequence $\left\{E_{n}\right\}$ has the properties that $E_{n} \uparrow I$ in $\mathscr{M}, T E_{n} \in$ $\langle\mathscr{M}\rangle$ for all $n$ and $\lim _{n \rightarrow \infty} T E_{n} x=T x$ for all $x \in X$. This shows that $T$ is a scalar-type spectral operator with respect to $\mathscr{M}$. Conversely, assume that $T$ is an everywhere defined scalar-type spectral operator. It follows from the definition that there exists a sequence $\left\{E_{n}\right\} \subseteq \mathscr{M}$ such that $T E_{n} \in\langle\mathscr{M}\rangle$ for all $n$ and $T E_{n} \rightarrow T$ strongly in $\mathscr{L}^{\#}(X)$. Hence, $T \in$ $\langle\mathscr{M}\rangle^{\#}$.

As observed in [4], Proposition 4.3, if $\mathscr{L}(X)$ is assumed to be sequentially complete, then $\langle\mathscr{M}\rangle^{\#}=\langle\mathscr{M}\rangle$. Combining these remarks we get the following result.

COROLlaRy 2.5. Let $\mathscr{M}$ be a Bade complete Boolean algebra of projections in the quasi-complete space $X$.

(i) $\langle\mathscr{M}\rangle^{\#}$ consists precisely of the everywhere defined scalar-type spectral operators with respect to $\mathscr{M}$.

(ii) If $\mathscr{L}(X)$ is assumed to be sequentially complete, then any everywhere defined scalar-type spectral operator with respect to $\mathscr{M}$, is continuous.

We denote by $\langle\mathscr{M}\rangle_{\infty}$ the collection of all scalar-type spectral operators with respect to $\mathscr{M}$. We show now that $\langle\mathscr{M}\rangle_{\infty}$ may be endowed with the structure of a commutative algebra, which extends that of $\langle\mathscr{M}\rangle$. As usual, if $S$ and $T$ are linear operators in $X$ with domain $\mathscr{D}(S)$ and $\mathscr{D}(T)$ respectively, then $\mathscr{D}(S+T)=\mathscr{D}(S) \cap \mathscr{D}(T)$ and $(S+T) x=S x+T x$ for all $x \in \mathscr{D}(S+T)$. Furthermore, $\mathscr{D}(S T)=\{x \in \mathscr{D}(T): T x \in \mathscr{D}(S)\}$ and $(S T) x=S(T x)$ for all $x \in \mathscr{D}(S T)$. We need the following preliminary result, the proof of which is straightforward and therefore omitted.

LEMMA 2.6. If $S$ and $T$ are scalar-type spectral operators with respect to $\mathcal{M}$, then $S+T$ and $S T$ are spectral elements.

We may now exhibit the algebraic structure on $\langle\mathscr{M}\rangle_{\infty}$.

Definition 2.7. If $S$ and $T$ are scalar-type spectral operators with respect to $\mathscr{M}$, then the scalar-type spectral operators $S+T$ and $S \cdot T$ are defined to be the maximal representatives of the corresponding classes $[S+T],[S T]$. 
It is useful to observe that, if $S, T, R \in\langle\mathscr{M}\rangle_{\infty}$ are given, then

(1) $S+T=R$ if and only if there exists a sequence $\left\{E_{n}\right\}$ in $\mathscr{M}$ which is determining for both $S$ and $T$, such that $E_{n}(X) \subseteq \mathscr{D}(R)$ and $S E_{n}+T E_{n}=R E_{n}$ for all $n$.

(2) $S \cdot T=R$ if and only if there exists a sequence $\left\{E_{n}\right\}$ in $\mathscr{M}$ which is determining for both $S$ and $T$, such that $E_{n}(X) \subseteq \mathscr{D}(R)$ and $\left(S E_{n}\right)\left(T E_{n}\right)=R E_{n}$ for all $n$.

The proof of the following result is now straightforward and accordingly the details are omitted.

Proposition 2.8. With respect to the above introduced operations, $\langle\mathscr{M}\rangle_{\infty}$ is a complex vector space and a commutative algebra with the identity operator as unit, which contains $\langle\mathscr{M}\rangle$ as a subalgebra.

3. $\langle\mathscr{M}\rangle_{\infty}$ as the universal completion of $\langle\mathscr{M}\rangle$. In this section we will show that the algebra $\langle\mathscr{M}\rangle_{\infty}$ of scalar-type spectral operators can be endowed with the structure of a universally complete unital $f$-algebra, which can be identified with the universal completion of the Dedekind complete unital $f$-algebra $\langle\mathscr{M}\rangle$.

We recall first that the (real) Riesz space $L$ is called laterally complete if every disjoint system in $L^{+}$has a supremum. If $L$ is Dedekind complete as well as laterally complete, then $L$ is called universally complete. Any Archimedean Riesz space $L$ has a (unique) universal completion $L^{u}$, i.e., $L^{u}$ is a universally complete Riesz space which contains $L$ as an order dense Riesz subspace (see e.g. [11], §50). If $L$ is Dedekind complete, then $L$ is an order ideal in $L^{u}$. The universal completion of a complex Riesz space is, by definition, the complexification of the universal completion of the real part of $L$.

If $L$ is a (complex) Dedekind complete Riesz space with universal completion $L^{u}$, then the Boolean algebra of band projections in $L^{u}$ is isomorphic (by restriction) to the Boolean algebra of band projections in $L$. Moreover, if we assume in addition that $L$ has a weak order unit $e$, then there exists for any $f \in L^{u}$ a sequence $\left\{P_{n}\right\}_{n=1}^{\infty}$ of band projections such that $P_{n} \uparrow I$ and such that $\left|P_{n} f\right| \leq n e$, and hence $P_{n} f \in L$, for all $n=1,2, \ldots$. In fact, we may take $P_{n}$ to be the projection onto the band generated by the element $(n e-|f|)^{+}$. Furthermore, if $L$ is an $f$-algebra with unit $e$, then the $f$-algebra multiplication in $L$ extends uniquely to an $f$-algebra multiplication in $L^{u}$, such that the element $e$ is the unit element in $L^{u}$. For details, see [15]. 
Suppose now that $\mathscr{M}$ is a Bade-complete Boolean algebra of projections in the quasi-complete space $X$. The strongly closed subalgebra $\langle\mathscr{M}\rangle$ of $\mathscr{L}(X)$ generated by $\mathscr{M}$, has the structure of a Dedekind complete (complex) $f$-algebra with unit element $I$. In the universal completion $\langle\mathscr{M}\rangle^{u}$ we consider the $f$-algebra structure which extends the structure of $\langle\mathscr{M}\rangle$.

Lemma 3.1. For every $T^{u} \in\langle\mathscr{M}\rangle^{u}$ there exists a unique element $T \in\langle\mathscr{M}\rangle_{\infty}$ such that for any $E \in \mathscr{M}$ with $T^{u} E \in\langle\mathscr{M}\rangle$, it follows that $E(X) \subseteq \mathscr{D}(T)$ and $T^{u} E=T E$.

(Remark: by $T^{u} E$ is meant the product of $T^{u}$ and $E$ in the universal completion $\langle\mathscr{M}\rangle^{u}$; by $T E$ is meant the composition of the operators $T$ and $E$.)

Proof. Suppose that $T^{u} \in\langle\mathscr{M}\rangle^{u}$. Observe first that if $x \in X$ and $E \in \mathscr{M}$ are such that $T^{u} E \in\langle\mathscr{M}\rangle$ and $E x=0$, then $\left(T^{u} E\right) x=\left(T^{u} E\right) E x$ $=0$. Since $T^{u} \in\langle\mathscr{M}\rangle^{u}$, there exists a sequence $\left\{E_{n}\right\} \subseteq \mathscr{M}$ with $E_{n} \uparrow I$ such that $T^{u} E_{n} \in\langle\mathscr{M}\rangle$ for all $n$. It follows from the above observation that if $x \in X, n \geq m$ and $x=E_{n} x=E_{m} x$, then $\left(T^{u} E_{n}\right) x=\left(T^{u} E_{m}\right) x$. We define the linear operator $T_{0}$ by setting $\mathscr{D}\left(T_{0}\right)=\bigcup_{n=1}^{\infty} E_{n}(X)$ and if $x \in \mathscr{D}\left(T_{0}\right)$, define $T_{0} x=\left(T^{u} E_{n}\right) x$ if $x=E_{n} x$. Note that $T_{0} E_{n}=T^{u} E_{n}$ for all $n$. It follows now that $T_{0}$ is well-defined and is a spectral element with determining sequence $\left\{E_{n}\right\}$. We define $T=\dot{T}_{0}$, the maximal extension of $T_{0}$, and note that $\left\{E_{n}\right\}$ is a determining sequence for $T$ (see Proposition 2.2). Suppose now that $E \in \mathscr{M}$ and $T^{u} E \in\langle\mathscr{M}\rangle$. If $x \in X$, then $\left(T_{0} E_{n}\right) E x=\left(T^{u} E_{n}\right) E x=\left(T^{u} E\right) E_{n} x$, which implies that $\left(T_{0} E_{n}\right) E x$ $\rightarrow\left(T^{u} E\right) x(n \rightarrow \infty)$, since $E_{n} x \rightarrow x(n \rightarrow \infty)$. Consequently $E(X) \subseteq$ $\mathscr{D}\left(\dot{T}_{0}\right)=\mathscr{D}(T)$ and $T E x=T^{u} E x$ for all $x \in X$.

Now assume that $S \in\langle\mathscr{M}\rangle_{\infty}$ has the property that $E(X) \subseteq \mathscr{D}(S)$ and $S E=T^{u} E$ whenever $E \in \mathscr{M}$ with $T^{u} E \in\langle\mathscr{M}\rangle$. This implies immediately that $E_{n}(X) \subseteq \mathscr{D}(S) \cap \mathscr{D}(T)$ and $S E_{n}=T E_{n}$ for all $n$, and so $S$ and $T$ are equivalent. Since $S$ and $T$ are both maximal, it follows that $S=T$ and the lemma is proved.

If $T^{u} \in\langle\mathscr{M}\rangle^{u}$, we denote by $\psi\left(T^{u}\right)$ the unique element of $\langle\mathscr{M}\rangle$ whose existence is shown in Lemma 3.1. Note that $\psi(T)=T$ for all $T \in\langle\mathscr{M}\rangle$. It follows from the proof of the above lemma that the sequence $\left\{E_{n}\right\}$ in $\mathscr{M}$ is a determining sequence for $\psi\left(T^{u}\right)$ whenever $E_{n} \uparrow I$ in $\mathscr{M}$ and $T^{u} E_{n} \in\langle\mathscr{M}\rangle$ for all $n$. We now show that $\langle\mathscr{M}\rangle_{\infty}$ may be identified with $\langle\mathscr{M}\rangle^{u}$. 
Proposition 3.2. The above defined mapping $\psi$ is an algebra isomorphism of the f-algebra $\langle\mathscr{M}\rangle^{u}$ onto the algebra $\langle\mathscr{M}\rangle_{\infty}$.

Proof. First of all, using the remark following Definition 2.7 in combination with the observation preceding this proposition, it is routine to show that $\psi$ is an algebra homomorphism. To see that $\psi$ is injective, suppose that $T^{u} \in\langle\mathscr{M}\rangle^{u}$ is such that $\psi\left(T^{u}\right)=0$. This implies that $T^{u} E=\psi\left(T^{u}\right) E=0$ for all $E \in \mathscr{M}$ with $T^{u} E \in\langle\mathscr{M}\rangle$, and since $\langle\mathscr{M}\rangle$ is an order dense ideal in $\langle\mathscr{M}\rangle^{u}$, we conclude that $T^{u}=0$. To show that $\psi$ is surjective, let $T \in\langle\mathscr{M}\rangle_{\infty}$ be given and let $\left\{E_{n}\right\} \subseteq \mathscr{M}$ be a determining sequence for $T$. Define $F_{1}=E_{1}$ and $F_{n}=E_{n}-E_{n-1}(n=2,3, \ldots)$. It is clear that $\left\{F_{n}\right\}$ is a disjoint sequence in $\mathscr{M}$ and $\sup _{n} F_{n}=I$. Moreover, $\left\{T F_{n}\right\}$ is a disjoint sequence in $\langle\mathscr{M}\rangle$. Now let $T^{u}=\sup _{n} T F_{n}$ in $\langle\mathscr{M}\rangle^{u}$, and note that $T^{u} F_{n}=T F_{n}$ for all $n$. Hence $T^{u} E_{n}=T E_{n}$, and so $\psi\left(T^{u}\right) E_{n}$ $=T^{u} E_{n}=T E_{n}$ for all $n$, which shows that $T=\psi\left(T^{u}\right)$. Therefore $\psi$ is a bijection of $\langle\mathscr{M}\rangle^{u}$ onto $\langle\mathscr{M}\rangle_{\infty}$, and by this the proof of the proposition is complete.

We observe that, if $\langle\mathscr{M}\rangle_{\infty}$ is given the partial ordering induced by the mapping $\psi$, then $\langle\mathscr{M}\rangle_{\infty}$ is a universally complete unital $f$-algebra containing $\langle\mathscr{M}\rangle$ as an order dense sub-algebra. From now on, we will identify the universal completion $\langle\mathscr{M}\rangle^{u}$ with $\langle\mathscr{M}\rangle_{\infty}$.

We conclude this section by observing that each element of $\langle\mathscr{M}\rangle_{\infty}$ has a familiar polar decomposition.

Proposition 3.3. If $S \in\langle\mathscr{M}\rangle_{\infty}$, then there exists $V \in\langle\mathscr{M}\rangle$ with $|V|=I$ such that $S=V \cdot|S|$ and $|S|=V^{-1} \cdot S$.

The proposition is an immediate consequence of the complex form of the Freudenthal spectral theorem and the fact that $Z\left(\langle\mathscr{M}\rangle_{\infty}\right)$ is precisely the order ideal generated by $I$ in $\langle\mathscr{M}\rangle$. See [5], Proposition 2.3 and [24], Theorem 141.1 for details. We note for future reference that an immediate consequence of Proposition 3.3 is the simple fact that $\mathscr{D}(S)=\mathscr{D}(|S|)$ for each $S \in\langle\mathscr{M}\rangle_{\infty}$.

4. Scalar-type spectral operators as extended orthomorphisms of cyclic subspaces. If $\mathscr{M}$ is an equicontinuous Bade complete Boolean algebra of projections in the quasi-complete space $X$, and if $x \in X$, then $\mathscr{M}(x)$ will denote the cyclic subspace generated by $x$. With the canonical order structure induced by the Boolean algebra $\mathscr{M}$, the space $\mathscr{M}(x)$ is a 
complex, Dedekind complete Riesz space, with a complete, locally solid Lebesgue topology. In this section, we will show that the restrictions to $\mathscr{M}(x)$ of the scalar-type spectral operators relative to $\mathscr{M}$ coincide with the extended orthomorphisms of the Riesz space $\mathscr{M}(x)$. We recall first some relevant facts from the theory of orthomorphisms. See, for example, [24] Chapter 20, and [9].

Let $L$ be an Archimedean (real) Riesz space. An extended orthomorphism in $L$ is an order bounded linear mapping $\pi$ from an order dense ideal $\mathscr{D}(\pi)$ in $L$ into $L$, with the property that $\pi f \perp g$ for all $f \in \mathscr{D}(\pi)$ and $g \in L$ with $f \perp g$. Each extended orthomorphism $\pi$ is order continuous, i.e., $u_{\tau} \downarrow 0$ in $\mathscr{D}(\pi)$ implies that $\inf _{\tau}\left|\pi u_{\tau}\right|=0$ in $L$ ([10], Theorem 1.3). By the same method as used in the proof of [24], Theorem 140.4, it follows that any extended orthomorphism $\pi$ can be written as $\pi=\pi^{+}$ $\pi^{-}$, where $\pi^{+}$and $\pi^{-}$are positive extended orthomorphisms with domain $\mathscr{D}(\pi)$, and $\pi^{+} u=(\pi u)^{+}, \pi^{-} u=(\pi u)^{-}$for all $0 \leq u \in \mathscr{D}(\pi)$. Furthermore, the absolute value of $\pi$ is defined by $|\pi|=\pi^{+}+\pi^{-}$. Note that $|\pi f|=|\pi| f||=|\pi|(|f|)$ for all $f \in \mathscr{D}(\pi)$. An extended orthomorphism $\pi$ such that $\mathscr{D}(\pi)=L$ is called, simply, an orthomorphism in $L$. Since extended orthomorphisms are order continuous, it follows that any extended orthomorphism $\pi$ has a unique maximal domain $\mathscr{D}^{m}(\pi)$. Two extended orthomorphisms are considered to be the same if they agree on some order dense ideal (equivalently, if their maximal extensions coincide). The set of all extended orthomorphisms in $L$ (with the above identification) is denoted by $\operatorname{Orth}^{\infty}(L)$, which is clearly a vector space with respect to the pointwise operations. A partial ordering in $\operatorname{Orth}^{\infty}(L)$ is defined by setting $\pi_{1} \leq \pi_{2}$ if $\pi_{1} u \leq \pi_{2} u$ for all $0 \leq u \in \mathscr{D}\left(\pi_{1}\right) \cap \mathscr{D}\left(\pi_{2}\right)$, and with respect to this partial ordering, $\operatorname{Orth}^{\infty}(L)$ is a Riesz space, such that $\left(\pi_{1} \vee \pi_{2}\right) u=\left(\pi_{1} u\right) \vee\left(\pi_{2} u\right)$ and $\left(\pi_{1} \wedge \pi_{2}\right) u=\left(\pi_{1} u\right) \wedge\left(\pi_{2} u\right)$ for all $0 \leq$ $u \in \mathscr{D}\left(\pi_{1}\right) \cap \mathscr{D}\left(\pi_{2}\right)$. Moreover, $\operatorname{Orth}^{\infty}(L)$ is laterally complete $([\mathbf{1 0}])$.

If $\pi_{1}, \pi_{2} \in \operatorname{Orth}^{\infty}(L)$, then $\mathscr{D}\left(\pi_{1} \pi_{2}\right)=\pi_{2}^{-1}\left(\mathscr{D}\left(\pi_{1}\right)\right)$ is an order dense ideal in $L$ ([10]), and the composition $\pi_{1} \cdot \pi_{2}$ is an extended orthomorphism. With respect to composition as multiplication, $\operatorname{Orth}^{\infty}(L)$ is an $f$-algebra with the identity operator as the unit element. The space of all orthomorphisms in $L$ is denoted by $\operatorname{Orth}(L)$, which is a subalgebra of $\operatorname{Orth}^{\infty}(L)$. If $L$ is Dedekind complete, then $\operatorname{Orth}^{\infty}(L)$ is Dedekind complete, hence universally complete, and $\operatorname{Orth}(L)$ is an order ideal in $\operatorname{Orth}^{\infty}(L)$.

All of the above results extend immediately to the complex setting, by means of complexification. We shall have need for the following simple characterization of the maximal domain of an extended orthomorphism. 
LEMMA 4.1. Let $L$ be a complex Dedekind complete Riesz space and let $\pi \in \operatorname{Orth}^{\infty}(L)$ with domain $\mathscr{D}(\pi)$ and maximal domain $\mathscr{D}^{m}(\pi)$. An element $f$ in $L$ belongs to $\mathscr{D}^{m}(\pi)$ if and only if the set $\{\pi g: g \in \mathscr{D}(\pi)$, $|g| \leq|f|\}$ is order bounded in L. In particular if $0 \leq \pi_{1} \leq \pi_{2}$ in $\operatorname{Orth}^{\infty}(L)$, then $\mathscr{D}^{m}\left(\pi_{2}\right) \subseteq \mathscr{D}^{m}\left(\pi_{1}\right)$ and $\mathscr{D}^{m}(|\pi|)=\mathscr{D}^{m}(\pi)$ for all $\pi \in \operatorname{Orth}^{\infty}(L)$.

Proof. First assume that $0 \leq \pi \in \operatorname{Orth}^{\infty}(L)$ with domain $\mathscr{D}(\pi)$, and let $\pi^{m}: \mathscr{D}^{m}(\pi) \rightarrow L$ be the maximal extension of $\pi$. Denote by $J$ the set of all $f \in L$ for which $\{\pi g: g \in \mathscr{D}(\pi),|g| \leq|f|\}$ is order bounded in $L$. Clearly, $J$ is an order ideal in $L$. If $f \in \mathscr{D}^{m}(\pi)$ and $g \in \mathscr{D}(\pi)$ with $|g| \leq|f|$, then $|\pi g|=\left|\pi^{m} g\right|=\left|\pi^{m}\right|(|g|) \leq\left|\pi^{m}\right|(|f|)$, which shows that $f \in$ $J$, and so $\mathscr{D}^{m}(\pi) \subseteq J$. For the proof of the converse inclusion, define for $0 \leq f \in J$

$$
\pi_{0} f=\sup \{\pi g: g \in \mathscr{D}(\pi), 0 \leq g \leq f\} .
$$

Note that, by the order continuity of $\pi$, we have $\pi_{0} f=\pi f$ for all $0 \leq f \in \mathscr{D}(\pi)$. Then $\pi_{0}$ is an additive mapping from $J^{+}$into $L^{+}$, which has an extension to a positive linear mapping from $J$ into $L$, which will be denoted by $\pi_{0}$ again. Clearly, $\pi_{0}$ is an extended orthomorphism, which extends $\pi$, and hence $J=\mathscr{D}\left(\pi_{0}\right) \subseteq \mathscr{D}^{m}(\pi)$.

At this point it is clear already that $0 \leq \pi_{1} \leq \pi_{2}$ in $\operatorname{Orth}^{\infty}(L)$ implies $\mathscr{D}^{m}\left(\pi_{2}\right) \subseteq \mathscr{D}^{m}\left(\pi_{1}\right)$, and $\mathscr{D}^{m}(|\pi|) \subseteq \mathscr{D}^{m}(\pi)$ for $\pi \in \operatorname{Orth}^{\infty}(L)$. Furthermore, a moment's reflection shows that $\mathscr{D}^{m}(\pi) \subseteq \mathscr{D}^{m}(|\pi|)$, hence $\mathscr{D}^{m}(|\pi|)$ $=\mathscr{D}^{m}(\pi)$ for all $\pi \in \operatorname{Orth}^{\infty}(L)$. This observation, combined with the first part of the proof, yields the result of the lemma.

Observe that it follows from the lemma that, if $\pi \in \operatorname{Orth}^{\infty}(L)$ and if $P$ is a band projection in $L$ such that $\pi P \in \operatorname{Orth}(L)$, then $P(L) \subseteq \mathscr{D}^{m}(\pi)$. Further, it follows from the above result, that if $0 \leq \pi_{\alpha} \uparrow_{\alpha} \pi$ holds in $\operatorname{Orth}^{\infty}(L)$, then $\mathscr{D}^{m}(\pi) \subseteq \bigcap_{\alpha} \mathscr{D}^{m}\left(\pi_{\alpha}\right)$ and if $0 \leq u \in \bigcap_{\alpha} \mathscr{D}^{m}\left(\pi_{\alpha}\right)$ is such that $\pi_{\alpha} u \uparrow_{\alpha} f$, then $u \in \mathscr{D}^{m}(\pi)$ and $\pi u=f$.

Now assume that $L$ is a locally solid Dedekind complete Riesz space with Lebesgue topology, and suppose $\pi \in \operatorname{Orth}^{\infty}(L)$ is given with domain $\mathscr{D}(\pi)$. We claim that $\mathscr{D}(\pi)=\mathscr{D}^{m}(\pi)$ if $\pi$ is closed. Indeed, since $\pi$ is closed if and only if $|\pi|$ is closed we may assume that $\pi \geq 0$. Now take $0 \leq f \in \mathscr{D}^{m}(\pi)$. Since $\mathscr{D}(\pi)$ is an order dense ideal in $L$, there exists a net $\left\{f_{\tau}\right\}$ in $\mathscr{D}(\pi)$ such that $0 \leq f_{\tau} \uparrow f$. By order continuity we have $0 \leq \pi f_{\tau}=\pi^{m} f_{\tau} \uparrow \pi^{m} f$, where $\pi^{m}$ denotes the maximal extension of $\pi$. Since the topology in $L$ is Lebesgue, this implies that $f_{\tau} \rightarrow f$ and $\pi f_{\tau} \rightarrow \pi^{m} f$ (topological convergence). Since $\pi$ is closed, this implies that $f \in \mathscr{D}(\pi)$, and hence $\mathscr{D}(\pi)=\mathscr{D}^{m}(\pi)$. 
Suppose now that $\mathscr{M}$ is an equicontinuous Bade complere Boolean algebra of projections in the quasi-complete space $X$. If $x \in X$, as above, we denote by $\mathscr{M}(x)$ the smallest closed $\mathscr{M}$-invariant subspace of $X$ containing $x$. With the canonical Riesz space structure induced by $\mathscr{M}$, $\mathscr{M}(x)$ is a complex, Dedekind complete Riesz space with a complete Lebesgue topology (see, for example, Proposition 3.9 of [4]). We gather some simple properties of the domains of scalar-type spectral operators.

LEMMA 4.2. Let $T$ be a scalar-type spectral operator with respect to $\mathscr{M}$, with domain $\mathscr{D}(T) \subseteq X$.

(i) If $x \in \mathscr{D}(T)$ and if $S \in\langle\mathscr{M}\rangle$, then $S x \in \mathscr{D}(T)$ and TSx $=S T x$.

(ii) If $x \in X$, then $\mathscr{D}(T) \cap \mathscr{M}(x)$ is an order dense ideal in the Riesz space $\mathscr{M}(x)$.

(iii) If $x \in X$, then $\mathscr{M}(x)$ is invariant under $T$, i.e., if $y \in \mathscr{D}(T) \cap$ $\mathscr{M}(x)$ then $T y \in \mathscr{M}(x)$.

Proof. (i) This follows simply from the $\mathscr{M}$-invariance of $\mathscr{D}(T)$ and the fact that $T$ is closed.

(ii) If $x \in X$ is given, suppose that $y, z \in \mathscr{M}(x)$ and that $|z| \leq|y|$ holds in $\mathscr{M}(x)$ with $y \in \mathscr{D}(T)$. By the Freudenthal spectral theorem, there exists $A \in Z(\mathscr{M}(x))$ such that $z=A y$. By Proposition 4.10 of [4], there exists $S \in\langle\mathscr{M}\rangle$ such that $A$ is the restriction of $S$ to $\mathscr{M}(x)$. It follows from part (i) that $z \in \mathscr{D}(T)$, and so $\mathscr{D}(T) \cap \mathscr{M}(x)$ is an order ideal in $\mathscr{M}(x)$. To see that $\mathscr{D}(T) \cap \mathscr{M}(x)$ is order dense in $\mathscr{M}(x)$ it suffices to note that if $0 \leq y \in \mathscr{M}(x)$ and if $\left\{E_{n}\right\} \subseteq \mathscr{M}$ is any determining sequence for $T$, then $E_{n} y \in \mathscr{D}(T) \cap \mathscr{M}(x)$ for all $n$ and $E_{n} y \uparrow y$. Part (iii) follows immediately from Corollary 2.4(iii).

If $T$ is any linear mapping in $X$ with domain $\mathscr{D}(T) \subseteq X$, and if $x \in X$ is such that $T$ leaves invariant $\mathscr{M}(x)$, then we denote by $T[x]$ the restriction of $T$ to $\mathscr{D}(T) \cap \mathscr{M}(x)$.

LeMma 4.3. If $T \in\langle\mathscr{M}\rangle_{\infty}$ has domain $\mathscr{D}(T)$ and if $x \in X$ then $T[x] \in \operatorname{Orth}^{\infty}(\mathscr{M}(x))$. Moreover, the maximal domain of $T[x]$ is precisely $\mathscr{D}(T) \cap \mathscr{M}(x)$.

Proof. Let $\left\{E_{n}\right\} \subseteq \mathscr{M}$ be any determining sequence for $T$. Suppose that $y, z \in \mathscr{D}(T) \cap \mathscr{M}(x)$ and that $|y| \leq|z|$ in $\mathscr{M}(x)$. Since $T E_{n} \in\langle\mathscr{M}\rangle$, it follows from Proposition 4.1 of [4] that $\left|T E_{n} y\right| \leq\left|T E_{n} z\right|$ for $n=1,2, \ldots$ Taking the limit as $n \rightarrow \infty$, we obtain $|T y| \leq|T z|$, which shows that $T[x]$ is order bounded. 
If now $y \in \mathscr{D}(T) \cap \mathscr{M}(x)$ and $z \in \mathscr{M}(x)$ such that $y \perp z$, then it follows once again from Proposition 4.1 of [4] that $T E_{n} y \perp z$ for all $n$, and consequently $T y \perp z$. Hence $T[x] \in \operatorname{Orth}^{\infty}(\mathscr{M}(x))$. Finally, since $T$ is closed, it is clear that $T[x]$ is closed as well, and so it follows from the remark following Lemma 4.1 that $\mathscr{D}(T) \cap \mathscr{M}(x)$ is the maximal domain of $T[x]$.

The carrier $E_{x} \in \mathscr{M}$ of $x \in X$ is defined by setting $E_{x}=\inf \{E \in \mathscr{M}$ : $E x=x\}$. The mapping $\Phi_{x}$ from $\langle\mathscr{M}\rangle_{\infty}$ into $\operatorname{Orth}^{\infty}(\mathscr{M}(x))$ is defined by setting $\Phi_{x}(T)=T[x]$. By the preceding lemma, it should be noted that $T[x]$ is in fact the maximal representative of the equivalence class it determines in $\operatorname{Orth}^{\infty}(\mathscr{M}(x))$. Further, for any $x \in X$ we denote $E_{x}\langle\mathscr{M}\rangle_{\infty}$ $=\left\{T E_{x}: T \in\langle\mathscr{M}\rangle_{\infty}\right\}$.

In the proof of the next result the following observation concerning orthomorphisms will be needed. Let $L$ be a (complex) Dedekind Riesz space, and take $0 \leq \pi \in \operatorname{Orth}^{\infty}(L)$. We assert that there exists a disjoint sequence $\left\{P_{n}\right\}_{n=1}^{\infty}$ of band projections in $L$ such that $\sup _{n} P_{n}=I$ and $0 \leq \pi P_{n} \leq n I$ (and hence $\left.\pi P_{n} \in Z(L)\right)$ for all $n$. Indeed, let $Q_{n}$ be the component of $I$ in the band $\left\{(n I-\pi)^{+}\right\}^{d d}$. Then $Q_{n} \uparrow I$ and $\pi Q_{n} \leq n I$ for all $n$. Defining $P_{1}=Q_{1}$ and $P_{n}=Q_{n}-Q_{n-1}$ for $n \geq 2$, the sequence $\left\{P_{n}\right\}$ has the desired properties.

Proposition 4.4. If $x \in X$, then the restriction map $\Phi_{x}: T \mapsto T[x]$ is an order continuous surjective f-algebra homomorphism from $\langle\mathscr{M}\rangle_{\infty}$ onto $\operatorname{Orth}^{\infty}(\mathscr{M}(x))$, which induces an f-algebra isomorphism from $E_{x}\langle\mathscr{M}\rangle_{\infty}$ onto $\operatorname{Orth}^{\infty}(\mathscr{M}(x))$.

Proof. It is not difficult to see that $\Phi_{x}$ is a positive algebra homomorphism from $\langle\mathscr{M}\rangle_{\infty}$ into $\operatorname{Orth}^{\infty}(\mathscr{M}(x))$, and consequently, $\Phi_{x}$ is a Riesz homomorphism.

To see that $\Phi_{x}$ is order continuous, suppose that $T_{0} \geq T_{\tau} \downarrow 0$ in $\langle\mathscr{M}\rangle_{\infty}$. It may be assumed that $T_{0} \geq I$, so that $T_{0}^{-1}$ exists in $\langle\mathscr{M}\rangle_{\infty}$, by [24] Theorem 146.3. It follows from the order continuity of the multiplication in the $f$-algebra $\langle\mathscr{M}\rangle_{\infty}$ that $I \geq T_{0}^{-1} T_{\tau} \downarrow 0$ in $\langle\mathscr{M}\rangle$. Since the restriction of $\Phi_{x}$ to $\langle\mathscr{M}\rangle$ is order continuous ([4], §4), we get $T_{0}^{-1} T_{\tau}[x] \downarrow 0$ in $Z(\mathscr{M}(x))$. Since $T_{\tau}[x]=T_{0}[x]\left(T_{0}^{-1} T_{\tau}\right)[x]$, the order continuity of the multiplication in $\operatorname{Orth}^{\infty}(\mathscr{M}(x))$ implies that $T_{\tau}[x] \downarrow 0$ in $\operatorname{Orth}^{\infty}(\mathscr{M}(x))$.

We show now that the mapping $\Phi_{x}$ is surjective. To this end, let $0 \leq A \in \operatorname{Orth}^{\infty}(\mathscr{M}(x))$ be given. By the remark preceding the proposition, there exists a sequence $\left\{P_{n}\right\}_{n=1}^{\infty}$ of band projections in the Riesz 
space $\mathscr{M}(x)$ such that $P_{n} \wedge P_{m}=0$ for $n \neq m, \sup _{n} P_{n}=I$ and $0 \leq A P_{n}$ $\leq n I$ in $\operatorname{Orth}^{\infty}(\mathscr{M}(x))$. Clearly, $\left\{A P_{n}\right\}$ is a disjoint sequence in $Z(\mathscr{M}(x))$ and $\sup _{n} A P_{n}=A$. By $\langle\mathscr{M}\rangle_{I}$ we denote the order ideal in $\langle\mathscr{M}\rangle_{\infty}$ generated by $I$, and let $E_{x}$ be the carrier projection of $x$. It follows from [4], Corollary 4.12 , that $\Phi_{x}$ induces an $f$-algebra isomorphism from $E_{x}\langle\mathscr{M}\rangle_{I}$ onto $Z(\mathscr{M}(x))$. Therefore, there exist $\left\{T_{n}\right\}_{n=1}^{\infty}$ in $\langle\mathscr{M}\rangle_{I}$ such that $0 \leq T_{n}$ $\leq n E_{x}$ for all $n$, and $T_{n} \wedge T_{m}=0$ for $n \neq m$ such that $\Phi_{x}\left(T_{n}\right)=T_{n}[x]=$ $A P_{n}$ for $n=1,2, \ldots$ Since $\langle\mathscr{M}\rangle_{\infty}$ is universally complete, there exists an element $T \in\langle\mathscr{M}\rangle_{\infty}$ such that $T=\sup _{n} T_{n}$. By the order continuity of $\Phi_{x}$ we find

$$
\begin{aligned}
\Phi_{x}(T) & =\Phi_{x}\left(\sup _{n} \sum_{k=1}^{n} T_{k}\right)=\sup _{n} \sum_{k=1}^{n} \Phi_{x}\left(T_{k}\right) \\
& =\sup _{n} \sum_{k=1}^{n} A P_{k}=\sup _{n} A P_{n}=A,
\end{aligned}
$$

which shows that $\Phi_{x}$ is surjective.

For the proof of the last statement of the proposition, let $N_{x}$ be the kernel of $\Phi_{x}$. Since $\Phi_{x}$ is an order continuous Riesz homomorphism, $N_{x}$ is a band, and so $\langle\mathscr{M}\rangle_{\infty}=N_{x} \oplus N_{x}^{d}$. By the above, $\Phi_{x}$ is surjective so $\Phi_{x}$ induces an isomorphism from $N_{x}^{d}$ onto $\operatorname{Orth}^{\infty}(\mathscr{M}(x))$. It is easily checked that $E_{x}$ is precisely the component of $I$ in $N_{x}^{d}$, and so $N_{x}^{d}=E_{x}\langle\mathscr{M}\rangle_{\infty}$. Therefore, $\Phi_{x}$ induces an isomorphism from $E_{x}\langle\mathscr{M}\rangle_{\infty}$ onto $\operatorname{Orth}^{\infty}(\mathscr{M}(x))$, and by this the proof of the proposition is complete.

We conclude this section with a result which generalizes Theorem 3.15 in Chapter XVII of [7]. See also [6], Proposition 2.2. First assume that $L$ is a Dedekind complete Riesz space with weak order unit $0 \leq e \in L$. We claim that for every $f \in L$ there exists a (unique) $\pi \in \operatorname{Orth}^{\infty}(L)$ such that $\pi e=f$. In fact, first note that we may restrict ourselves to the case that $f \geq 0$. Let $u_{n}$ be the component of $f$ in the band generated by $(n e-f)^{+}$ for $n=1,2, \ldots$, then $0 \leq u_{n} \uparrow_{n} f$ and $0 \leq u_{n} \leq n e$ for all $n$. Now define $w_{1}=u_{1}$ and $w_{n}=u_{n}-u_{n-1}$ for $n \geq 2$, then $\left\{w_{n}\right\}_{n=1}^{\infty}$ is a disjoint sequence in $L$ such that $0 \leq w_{n} \leq n e$ for all $n$ and $f=\sup _{n} w_{n}$. By the Freudenthal spectral theorem, for each $n$ there exists $\pi_{n} \in Z(L)$ such that $\pi_{n} e=w_{n}$ and $0 \leq \pi_{n} \leq n I$. Since $\left\{w_{n}\right\}$ is a disjoint sequence, we can assume that $\left\{\pi_{n}\right\}$ is a disjoint sequence as well. Since $\operatorname{Orth}^{\infty}(L)$ is universally complete, $\pi=\sup _{n} \pi_{n}$ exists in $\operatorname{Orth}^{\infty}(L)$. Since $\sup _{n} \pi_{n} e=$ $\sup _{n} w_{n}=f \in L$, it follows from the remark following Lemma 4.1, that $e \in \mathscr{D}(\pi)$ and $\pi e=f$, where $\mathscr{D}(\pi)$ denotes the maximal domain of $\pi$. Combining this observation with the result of the preceding proposition, we obtain the following result. 
Proposition 4.5. Let $\mathscr{M}$ be an equicontinuous Bade complete Boolean algebra of projections in the quasi-complete space $X$. For $x \in X$ and $y \in \mathscr{M}(x)$ there exists $T \in\langle\mathscr{M}\rangle_{\infty}$ such that $x \in \mathscr{D}(T)$ and $T x=y$. Hence, $\mathscr{M}(x)=\left\{T x: T \in\langle\mathscr{M}\rangle_{\infty}\right.$ and $\left.x \in \mathscr{D}(T)\right\}$.

5. Reflexivity of scalar-type spectral operators. Let $\mathscr{M}$ be an equicontinuous Bade complete Boolean algebra of projections in the quasi-complete space $X$. As before, we denote by $\langle\mathscr{M}\rangle$ the strongly closed subalgebra of $\mathscr{L}(X)$ generated by $\mathscr{M}$, in which we consider the canonical Riesz space structure. By $\langle\mathscr{M}\rangle_{I}$ we denote the order ideal generated by $I$ in $\langle\mathscr{M}\rangle$. Let $T$ be a densely defined linear operator in $X$ with $\langle\mathscr{M}\rangle_{r}$-invariant domain $\mathscr{D}(T)$. Suppose that $T$ leaves invarant each $\mathscr{M}$-invariant subspace of $X$, i.e., if $Y \subseteq X$ is a closed, $\mathscr{M}$-invariant subspace, then $T(\mathscr{D}(T) \cap Y) \subseteq Y$. In particular, if $E \in \mathscr{M}$, then $T(\mathscr{D}(T) \cap E(X)) \subseteq$ $E(X)$. It follows routinely that $T E x=E T x$ for all $x \in \mathscr{D}(T)$ and $E \in \mathscr{M}$. Note that we do not assume that $T$ is closed.

If $x \in X$, then we denote by $T[x]$ the restriction of $T$ to $\mathscr{D}(T) \cap$ $\mathscr{M}(x)$, so $T[x]$ is a linear operator in $\mathscr{M}(x)$ with domain $\mathscr{D}(T) \cap \mathscr{M}(x)$. In the next proposition we collect some properties of $T[x]$.

Proposition 5.1. If $T$ is a densely defined linear operator in $X$ with $\langle\mathscr{M}\rangle_{\Gamma}$-invariant domain, if $T$ leaves invariant each $\mathscr{M}$-invariant closed subspace of $X$ and if $x \in X$, then

(i) $\mathscr{D}(T) \cap \mathscr{M}(x)$ is an order ideal in $\mathscr{M}(x)$.

(ii) $T[x]$ is a band preserving operator.

Proof. (i) Suppose $y, z \in \mathscr{M}(x)$ and $|y| \leq|z|, z \in \mathscr{D}(T) \cap \mathscr{M}(x)$. As in the proof of Lemma 4.2(ii), there exists $S \in\langle\mathscr{M}\rangle_{I}$, such that $y=S z$. Since $\mathscr{D}(T)$ is $\langle\mathscr{M}\rangle_{r}$ invariant, it follows that $y \in \mathscr{D}(T) \cap \mathscr{M}(x)$.

(ii) Now suppose that $y \in \mathscr{D}(T) \cap \mathscr{M}(x)$ and $z \in \mathscr{M}(x)$ such that $y \perp z$. Then there exists $E \in \mathscr{M}$ such that $E y=y$ and $E z=0$. Since $y \in \mathscr{D}(T)$, it follows that $T y=T E y=E T y$, so $E(T y)=T y$ and $E z=0$, which shows that $T y \perp z$.

Our next objective is to show that $T[x]$ is in fact an order bounded operator from $\mathscr{D}(T) \cap \mathscr{M}(x)$ into $\mathscr{M}(x)$. For this purpose we make some remarks concerning band preserving operators. Let $L$ be an Archimedean Riesz space and let $A$ be an ideal in $L$. Suppose that $T$ is a band preserving operator from $A$ into $L$. A straightforward modification of the proof of [14], Proposition 6 shows that if $T$ is order bounded on some order dense ideal in $A$, then $T$ is order bounded on $A$. 
LEMMA 5.2. Let L be a uniformly complete Riesz space with a separating family of order continuous linear functionals. Suppose that $A$ is an ideal in $L$ and that $T$ is a band preserving operator from $A$ into $L$. Then $T$ is order bounded.

Proof. By the above remark, it is sufficient to prove that $T$ is order bounded on some order dense ideal in $A$. As in the proof of [4], Lemma 2.6, we may restrict ourselves to the situation that there exists a strictly positive order continuous linear functional. It is then clear that for any positive disjoint sequence $\left\{w_{n}\right\}_{n=1}^{\infty}$ in $L$ there exist real numbers $\lambda_{n}>0$ $(n=1,2, \ldots)$ such that $\left\{\lambda_{n} w_{n}\right\}_{n=1}^{\infty}$ is not order bounded in $L$. Considering $T$ as a disjointness preserving operator from $A$ into $L$, we can apply [14], Theorem 8, which shows that $T$ is order bounded on some order dense ideal in $A$.

We now return to the situation of Proposition 5.1. Applying the above observations to the operator $T[x]$ we get the following result.

Proposition 5.3. Let $T$ be as in Proposition 5.1. For each $x \in X$ the operator $T[x]$ is band preserving and order bounded from the ideal $\mathscr{D}(T) \cap$ $\mathscr{M}(x)$ into $\mathscr{M}(x)$. If $x \in X$ is such that $\mathscr{D}(T) \cap \mathscr{M}(x)$ is order dense in $\mathscr{M}(x)$, then $T[x] \in \operatorname{Orth}^{\infty}(\mathscr{M}(x))$. In particular, $T[x] \in \operatorname{Orth}^{\infty}(\mathscr{M}(x))$ for all $x \in \mathscr{D}(T)$.

Proof. Since $\mathscr{M}(x)$ is a locally convex solid Riesz space with Lebesgue topology, and since $T[x]$ is band preserving (by Proposition 5.1), it follows from Lemma 5.2 that $T[x]$ is order bounded. Therefore, by definition, $T[x] \in \operatorname{Orth}^{\infty}(\mathscr{M}(x))$ whenever $\mathscr{D}(T) \cap \mathscr{M}(x)$ is order dense in $\mathscr{M}(x)$. If $x \in \mathscr{D}(T)$, then $\mathscr{D}(T) \cap \mathscr{M}(x)$ is order dense in $\mathscr{M}(x)$, as $x$ is a weak order unit in $\mathscr{M}(x)$.

We remark that it follows from the above proposition that, if $x \in$ $\mathscr{D}(T)$ and $S \in\langle\mathscr{M}\rangle_{I}$, then $S T x=T S x$. In fact, if $x \in \mathscr{D}(T)$, then $T[x] \in \operatorname{Orth}^{\infty}(\mathscr{M}(x))$. and $S[x]$ is an element of $Z(\mathscr{M}(x)) \subseteq$ $\operatorname{Orth}^{\infty}(\mathscr{M}(x))$, which implies that $T[x]$ and $S[x]$ commute. Note that, if $x \in \mathscr{D}(T)$, then $\mathscr{D}(T) \cap \mathscr{M}(x)$ is, in general, not the maximal domain of $T[x]$, since we do not assume that $T$ is closed.

For any $E \in \mathscr{M}$, the operator $T E$ is defined by $T E x=T(E x)$ for all $x \in \mathscr{D}(T E)=E^{-1}(\mathscr{D}(T))$. Note that $\mathscr{D}(T) \subseteq \mathscr{D}(T E)$, so $T E$ is densely defined. An appropriate modification of the proof of Lemma 5.3 in [4] yields the following result. 
LeMma 5.4. Suppose that $T$ is as above and $x \in \mathscr{D}(T)$. If $T x=0$, then $T E_{x} \subseteq 0$, where $E_{x}$ is the carrier projection of $x$ in $\mathscr{M}$.

Proposition 5.5. Let $T$ be a densely defined linear operator in $X$ with

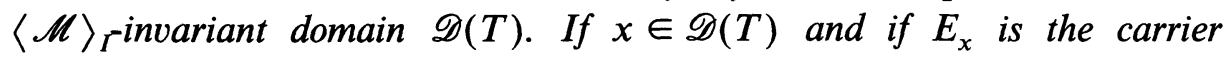
projection of $x$ in $\mathscr{M}$, then there exists $S \in E_{x}\langle\mathscr{M}\rangle_{\infty}$ such that $T E_{x} \subseteq S$.

Proof. Since $x \in \mathscr{D}(T)$, it follows from Proposition 5.3 that $T[x] \in$ $\operatorname{Orth}^{\infty}(\mathscr{M}(x))$. Therefore, by Proposition 4.4 , there exists a (unique) $S \in E_{x}\langle\mathscr{M}\rangle_{\infty}$ such that $T[x] \subseteq S[x]$. We assert that $T E_{x} \subseteq S$. Indeed, let $\left\{E_{n}\right\}_{n=1}^{\infty}$ be a determining sequence for $S$, and define the operators $R_{n}=S E_{n}-T E_{n}$ with domain $\mathscr{D}\left(R_{n}\right)=\mathscr{D}\left(T E_{n}\right)=\left\{y \in X: \quad E_{n} y \in\right.$ $\mathscr{D}(T)\}$ for $n=1,2, \ldots$. Since $\mathscr{D}(T) \subseteq \mathscr{D}\left(R_{n}\right)$, it follows that $R_{n}$ is densely defined. Furthermore, $\mathscr{D}\left(R_{n}\right)$ is $\langle\mathscr{M}\rangle_{r}$ invariant and $R_{n}$ leaves invariant all $\mathscr{M}$-invariant closed subspaces of $X$. Moreover, $x \in \mathscr{D}\left(R_{n}\right)$ and $R_{n} x=S E_{n} x-T E_{n} x=S[x] E_{n} x-T[x] E_{n} x=0$, and so it follows from Lemma 5.4 that $R_{n} E_{x} \subseteq 0$. We thus have shown that $T E_{n} E_{x} \subseteq$ $S E_{n} E_{x}$, i.e., that $T E_{n} E_{x} \subseteq S E_{n}$ for all $n$. Now take $y \in \mathscr{D}\left(T E_{x}\right)$ i.e., $E_{x} y \in \mathscr{D}(T)$. Then $E_{n} y \rightarrow y$ as $E_{n} \uparrow I$ in $\mathscr{M}$, and

$$
S E_{n} y=T E_{n} E_{x} y=T E_{x} E_{n} y=E_{n}\left(T E_{x} y\right) \rightarrow T E_{x} y .
$$

Hence, since $S$ is closed, $y \in \mathscr{D}(S)$ and $S y=T E_{x} y$. This shows that $T E_{x} \subseteq S$, and the proposition is completely proved.

LEMMA 5.6. Let $T$ be a densely defined linear operator with $\langle\mathscr{M}\rangle_{r}$-invariant domain $\mathscr{D}(T)$. Assume that $T$ leaves invariant each $\mathscr{M}$-invariant closed subspace of $X$. There exists a family $\left\{x_{\alpha}\right\} \subseteq \mathscr{D}(T)$ with mutually disjoint carrier projections $\left\{E_{\alpha}\right\} \subseteq \mathscr{M}$, such that $\sup _{\alpha} E_{\alpha}=I$.

Proof. By Zorn's lemma, there exists a maximal disjoint system $\left\{E_{\alpha}\right\}$ of carrier projections in $\mathscr{M}$ corresponding to elements $\left\{x_{\alpha}\right\}$ in $\mathscr{D}(T)$. Set $E_{0}=\sup E_{\alpha}$ in $\mathscr{M}$ and suppose that $E_{0} \neq I$. Let $F_{0}=I-E_{0}$, then $F_{0} \neq 0$. Since $\mathscr{D}(T)$ is dense in $X$, there exists $y \in \mathscr{D}(T)$ such that $F_{0} y \neq 0$. If $z=F_{0} y$, then $z \neq 0$ and $z \in \mathscr{D}(T)$. The carrier projection $E_{z}$ of $z$ satisfies $E_{z} \neq 0$ and $E_{z} \leq F_{0}$, as $F_{0} z=z$. Hence $E_{z} \perp E_{0}$, contradicting the maximality of the system $\left\{E_{\alpha}\right\}$.

We are finally in a position to prove the main result of this paper.

THEOREM 5.7. Let $\mathscr{M}$ be an equicontinuous Bade complete Boolean algebra of projections in the quasi-complete space $X$. Let $T$ be a densely 
defined linear operator in $X$ with $\langle\mathscr{M}\rangle_{r^{-i n v a r i a n t}}$ domain $\mathscr{D}(T)$. The following statements are equivalent.

(i) $T$ leaves invariant all $\mathscr{M}$-invariant closed subspaces of $X$.

(ii) $T$ is closable and its closure $\tilde{T}$ belongs to $\langle\mathscr{M}\rangle_{\infty}$.

Proof. (ii) $\Rightarrow$ (i). If $\tilde{T} \in\langle\mathscr{M}\rangle_{\infty}$, then, by Corollary 2.4, $\tilde{T}$ leaves invariant all $\mathscr{M}$-invariant closed subspaces of $X$, and since $T \subseteq \tilde{T}$, we conclude that $T$ has the same property.

(i) $\Rightarrow$ (ii). Now suppose that $T$ leaves invariant all $\mathscr{M}$-invariant closed subspaces of $X$. By Lemma 5.6, there exists a disjoint system of carrier projections $\left\{E_{\alpha}: \alpha \in \mathscr{Q}^{\prime}\right\}$ in $\mathscr{M}$, corresponding to elements $\left\{x_{\alpha}: \alpha \in \mathscr{Q}\right\}$ in $\mathscr{D}(T)$, with $\sup _{\alpha} E_{\alpha}=I$. By Proposition 5.5, for each $\alpha$ there exists $S_{\alpha} \in E_{\alpha}\langle\mathscr{M}\rangle$ such that $T E_{\alpha} \subseteq S_{\alpha}$. Then $\left\{S_{\alpha}: \alpha \in \mathscr{Q}\right\}$ is a disjoint system in $\langle\mathscr{M}\rangle_{\infty}$, and since $\langle\mathscr{M}\rangle_{\infty}$ is laterally complete, there exists a unique element $S \in\langle\mathscr{M}\rangle_{\infty}$ such that $S E_{\alpha}=S_{\alpha}$ for all $\alpha$. We will show now that $T \subseteq S$. To this end, we define for every finite subset $\mathscr{F}$ of $\mathscr{2}$ the projection $E_{\mathscr{F}}=\sum_{\alpha \in \mathscr{F}} E_{\alpha}$. Clearly, $E_{\mathscr{F}} \uparrow I$ in $\mathscr{M}$. Take $x \in \mathscr{D}(T)$, then $E_{\mathscr{F}} x \rightarrow x$ and $x \in \mathscr{D}\left(T E_{\alpha}\right)$ for all $\alpha \in \mathscr{Q}$. Hence, $x \in \mathscr{D}\left(S_{\alpha}\right)$ and $S_{\alpha} x=$ $T E_{\alpha} x$ for all $\alpha$. Therefore, $E_{\mathscr{F}} x \in \mathscr{D}(S)$ for all finite subsets $\mathscr{F}$ of $\mathscr{Q}$ and

$$
S E_{\mathscr{F}} x=T E_{\mathscr{F}} x=E_{\mathscr{F}} T x \rightarrow T x .
$$

Since $S$ is closed, this implies that $x \in \mathscr{D}(S)$ and $S x=T x$, which shows that $T \subseteq S$.

It follows from the inclusion $T \subseteq S$ and from the closedness of $S$, that $T$ is closable. Let $\tilde{T}$ denote the closure of $T$, so $T \subseteq \tilde{T} \subseteq S$. We claim that $\tilde{T}=S$. First note that $\mathscr{D}(\tilde{T})$ is $\mathscr{M}$-invariant. Indeed, suppose that $\tilde{x} \in \mathscr{D}(\tilde{T})$ and $E \in \mathscr{M}$. Then there exists a net $\left\{x_{\alpha}\right\}$ in $\mathscr{D}(T)$ such that $x_{\alpha} \rightarrow \tilde{x}$ and $T x_{\alpha} \rightarrow \tilde{T} \tilde{x}$. Hence, $E x_{\alpha} \rightarrow E \tilde{x}$ and $T E x_{\alpha}=E T x_{\alpha} \rightarrow E \tilde{T} \tilde{x}$, which shows that $E \tilde{x} \in \mathscr{D}(\tilde{T})$ and $\tilde{T} E \tilde{x}=E \tilde{T} \tilde{x}$. This implies that $\mathscr{D}(\tilde{T})$ $\subseteq \mathscr{D}(\tilde{T} E)$, and hence $\mathscr{D}(\tilde{T} E)$ is dense, for all $E \in \mathscr{M}$. Now let $\left\{E_{n}\right\}$ be a determining sequence for $S$ in $\mathscr{M}$. Clearly $\tilde{T} E_{n} \subseteq S E_{n}$ for all $n=1,2, \ldots$ Since $\tilde{T} E_{n}$ is closed and densely defined, and since $S E_{n}$ is continuous, it follows that $\tilde{T} E_{n}=S E_{n}$ for all $n$. Now take $x \in \mathscr{D}(S)$, then $S E_{n} x \rightarrow S x$. We thus have $E_{n} x \rightarrow x, E_{n} x \in \mathscr{D}(\tilde{T})$ and $\tilde{T} E_{n} x=S E_{n} x \rightarrow S x$. Since $\tilde{T}$ is closed we may conclude that $x \in \mathscr{D}(\tilde{T})$ and $\tilde{T} x=S x$. This shows that $S \subseteq \tilde{T}$, and hence $\tilde{T}=S \in\langle\mathscr{M}\rangle_{\infty}$, by which the theorem is proved.

Note that, by the observation in Corollary 2.5(i), the above theorem includes the result of Theorem 5.5 in [4]. 
Corollary 5.8. Let $\mathscr{M}$ be an equicontinuous Bade complete Boolean algebra of projections in the quasi-complete space $X$. Let $T$ be a densely defined, closed linear operator in X with $\mathscr{M}$-invariant domain $\mathscr{D}(T)$. The following statements are equivalent.

(i) T leaves invariant all $\mathscr{M}$-invariant subspaces of $X$.

(ii) $T \in\langle\mathscr{M}\rangle_{\infty}$, i.e., $T$ is a scalar-type spectral operator with respect to $\mathscr{M}$.

Proof. Only the implication (i) $\Rightarrow$ (ii) needs a proof. By the above theorem it is sufficient to show that $\mathscr{D}(T)$ is in fact $\langle\mathscr{M}\rangle_{\Gamma}$-invariant. To this end, first observe that $T E x=E T x$ for all $x \in \mathscr{D}(T)$ and $E \in \mathscr{M}$ (same argument as at the beginning of the present section). Let $M$ be the linear subspace of $\mathscr{L}(X)$ generated by $\mathscr{M}$. Clearly, $\mathscr{D}(T)$ is $M$-invariant and $T S x=S T x$ for all $x \in \mathscr{D}(T)$ and $S \in M$. Now take $x \in \mathscr{D}(T)$ and $R \in\langle\mathscr{M}\rangle_{I}$. Then there exists a net $\left\{S_{\alpha}\right\} \subseteq M$ such that $S_{\alpha} \rightarrow R$ strongly. Hence $S_{\alpha} x \in \mathscr{D}(T)$ for all $\alpha, S_{\alpha} x \rightarrow R x$ and $T S_{\alpha} x=S_{\alpha} T x \rightarrow R T x$. Since $T$ is closed, this implies that $R x \in \mathscr{D}(T)$, and we are done.

We end this section with an example which shows that in Theorem 5.7 the condition that $\mathscr{D}(T)$ is $\langle\mathscr{M}\rangle_{I}$ invariant cannot be weakened to $\mathscr{M}$-invariance of $\mathscr{D}(T)$.

EXAMPLE 5.9. Consider the unit interval $[0,1]$ with Lebesgue measure, let $X$ be the Banach space $L_{p}[0,1](1 \leq p<\infty)$ and let $\mathscr{M}$ be the Boolean algebra of all band projections in $L_{p}[0,1]$. Note that the projections in $\mathscr{M}$ are precisely the multiplications by characteristic functions of measurable subsets of $[0,1]$. Then $\mathscr{M}$ is equicontinuous and Bade complete. The $\mathscr{M}$-invariant closed subspaces of $L_{p}[0,1]$ are precisely the bands (cf. [5], Proposition 2.4). Furthermore, $\langle\mathscr{M}\rangle=\langle\mathscr{M}\rangle_{I}$ can be identified with $L_{\infty}[0,1]$, acting on $L_{p}[0,1]$ by multiplication. Let $\mathscr{D}(T)$ be the subspace of $L_{p}[0,1]$ consisting of all functions which can be written as $f=\sum_{i=1}^{n} p_{i} \chi_{E_{i}}$, where $E_{1}, \ldots, E_{n}$ are disjoint measurable subsets of $[0,1]$, and $p_{1}(x), \ldots, p_{n}(x)$ are linear functions. Clearly, $\mathscr{D}(T)$ is dense in $L_{p}[0,1]$ and $\mathscr{M}$-invariant, but $\mathscr{D}(T)$ is not $\langle\mathscr{M}\rangle_{r}$ invariant. For $f=$ $\sum_{i=1}^{n} p_{i} \chi_{E_{i}}$ in $\mathscr{D}(T)$, define $T f=\sum_{i=1}^{n} p_{i}^{\prime} \chi_{E_{i}}$, where $p_{i}^{\prime}$ denotes the derivative of $p_{i}$. Clearly $T$ is a linear operator from $\mathscr{D}(T)$ into $L_{p}[0,1]$, which leaves invariant all $\mathscr{M}$-invariant closed subspaces of $L_{p}[0,1]$. Now it is not difficult to find a sequence $\left\{f_{n}\right\}_{n=1}^{\infty}$ in $\mathscr{D}(T)$ such that $f_{n} \rightarrow 0$ (norm) and $T f_{n}=1$ for all $n$, which shows that $T$ is not closable. 
We will indicate in the next section that the preceding Theorem 5.7 and Corollary 5.8 extend results of Masani and Rosenberg [13], Rosenthal and Sourour [18], [19] and Sourour [21], [22]. Moreover, even in the case that $X$ is Banach, the result of Theorem 5.1 appears to be new.

6. The spectrum of a scalar-type spectral operator. We begin by showing that if $\mathscr{M}$ is a Bade complete Boolean algebra of projections in $X$ and if $T$ is scalar-type spectral with respect to $\mathscr{M}$ i.e. if $T \in\langle\mathscr{M}\rangle_{\infty}$, then $T$ is scalar-type spectral in the sense of Dunford. Conversely, if $T$ is scalar-type spectral in the sense of Dunford, then there exists a Bade complete Boolean algebra of projections $\mathscr{M}$ for which $T \in\langle\mathscr{M}\rangle_{\infty}$.

A spectral measure in $X$ is a countably additive map $E: \mathscr{B} \rightarrow \mathscr{L}(X)$ whose domain $\mathscr{B}$ is a $\sigma$-algebra of subsets of some set $\Omega$, which is multiplicative and satisfies $E(\Omega)=I$. The spectral measure $E$ is called equicontinuous if the range of $E$ is an equicontinuous subset of $\mathscr{L}(X)$ and closed if its range is a Bade complete Boolean algebra of projections in $X$. If $E: \mathscr{B} \rightarrow \mathscr{L}(X)$ is a spectral measure and $f$ a complex valued $\mathscr{B}$-measurable function, then $f$ is said to be $E$-integrable if $f$ is integrable with respect to the complex measure $\left\langle E x, x^{\prime}\right\rangle$ for each $x \in X, x^{\prime} \in X^{\prime}$ and there exists an operator, denoted $\int_{\Omega} f d E$, in $\mathscr{L}^{\#}(X)$ such that

$$
\left\langle\left(\int_{\Omega} f d E\right) x, x^{\prime}\right\rangle=\int_{\Omega} f d\left\langle E x, x^{\prime}\right\rangle
$$

for each $x \in X, x^{\prime} \in X^{\prime}$.

The class $\mathscr{L}^{1}(E)$ of all $E$-integrable complex functions on $\Omega$ is a Riesz space for the pointwise ordering on $\Omega$, containing all bounded $\mathscr{B}$-measurable functions. The $\mathscr{B}$-measurable function $f$ on $\Omega$ is said to be $E$-null if $f$ is $E$-integrable and $\int_{\Omega} f d E=0$. The class of complex $E$-null functions on $\Omega$ is an order ideal in $\mathscr{L}^{1}(E)$ and the corresponding quotient space is denoted by $L^{1}(E)$. If $E$ is closed, then the map $f \rightarrow \int_{\Omega} f d E$ is an order isomorphism of $L^{1}(E)$ onto $\langle\mathscr{M}\rangle^{\#}$ where $\mathscr{M}$ is the closure of the range of $E$ in $\mathscr{L}(X)$. If now $f$ is a $\mathscr{B}$-measurable complex valued function on $\Omega$, the spectral integral $\int_{\Omega} f d E$ is defined as follows. If $\left\{B_{n}\right\}$ is any sequence of $\mathscr{B}$-measurable subsets of $\Omega$ such that $\chi_{B_{n}} \uparrow_{n} \chi_{\Omega}$ in $L^{1}(E)$ and $f \chi_{B_{n}} \in L^{1}(E), n=1,2, \ldots$, then $x \in \mathscr{D}\left(\int_{\Omega} f d E\right)$ if and only if $\lim _{n \rightarrow \infty}\left(\int f \chi_{B_{n}} d E\right)(x)$ exists in $X$, in which case $\left(\int_{\Omega} f d E\right)(x)=$ $\lim _{n}\left(\int_{\Omega} f \chi_{B_{n}} d E\right)(x)$. The linear mapping $T$ in $X$ is called a scalar-type spectral operator in the sense of Dunford if there exists an equicontinuous spectral measure $E: \mathscr{B} \rightarrow \mathscr{L}(X)$ and a $\mathscr{B}$-measurable complex function $f$ such that $T=\int_{\Omega} f d E$. We remark that if $\mathscr{L}(X)$ is sequentially complete 
and if $T$ is scalar-type spectral in the sense of Dunford then $T \in \mathscr{L}(X)$ if $\mathscr{D}(T)=X$. For example, see [5] Proposition 1.2.

Suppose now that $T$ is a scalar-type spectral operator in $X$ in the sense of Dunford, with representation as a spectral integral given by $\int_{\Omega} f d E$, with $E: \mathscr{B} \rightarrow \mathscr{L}(X)$ an equicontinuous spectral measure. If $\mathscr{M}$ denotes the closure in $\mathscr{L}(X)$ of the range of the spectral measure $E$, then $\mathscr{M}$ is a Bade complete Boolean algebra of projections in $X$ and it follows from Definition 2.3 above and [6], Proposition 1.8 that $T$ is a scalar-type spectral operator with respect to $\mathscr{M}$, i.e. that $T$ is an element of $\langle\mathscr{M}\rangle_{\infty}$. Conversely, if $\mathscr{M}$ is a Bade complete Boolean algebra of projections in $X$, and if $T \in\langle\mathscr{M}\rangle_{\infty}$ then it is a consequence of the Freudenthal spectral theorem that there is an equicontinuous spectral measure $E$ defined on the Borel subsets of the complex plane $\mathbf{C}$ such that $\int_{\mathbf{C}} z d E$ is a representation of $T$ as a spectral integral. In fact, we may write $T=\operatorname{Re} T+i \operatorname{Im} T$ with $\operatorname{Re} T, \operatorname{Im} T \in \operatorname{Re}\langle\mathscr{M}\rangle_{\infty}$. Denote by $\{F(\lambda): \lambda \in \mathbf{R}\},\{G(\lambda): \lambda \in \mathbf{R}\}$ respectively the Freudenthal spectral systems $([11], \S \S 38,40)$ of $\operatorname{Re} T, \operatorname{Im} T$ in the Dedekind complete (real) Riesz space $\operatorname{Re}\langle\mathscr{M}\rangle_{\infty}$ with respect to the weak order unit $I$. The Freudenthal system $\{E(z): z \in \mathbf{C}\}$ of $T$ in $\mathscr{M}$ is then defined by setting $E(z)=F(\lambda) G(\mu)$ if $z=\lambda+i \mu, \lambda, \mu \in \mathbf{R}$. The spectral system $\{E(z): z \in \mathbf{C}\}$ induces, in the usual way, a countably additive, multiplicative $\mathscr{M}$-valued set function $E_{0}$ on the ring generated by the collection of all half-open cells in $\mathbf{C}$ of the form

$$
\left[z_{1}, z_{2}\right)=\left\{x+i y \in \mathbf{C}: \operatorname{Re} z_{1} \leq x<\operatorname{Re} z_{2}, \operatorname{Im} z_{1} \leq y<\operatorname{Im} z_{2}\right\} .
$$

Since $\langle\mathscr{M}\rangle$ is Dedekind complete and has Lebesgue topology, it follows from the Kluvánek extension theorem ([8] p. 118; see also [23] Chapter 11) that $E_{0}$ extends to a countably additive measure, which we denote by $E$, on the Borel subsets of $\mathbf{C}$. As in Proposition 3.6 of [6], it follows that $E$ is an $\mathscr{M}$-valued equicontinuous spectral measure. From the Freudenthal spectral theorem and the dominated convergence theorem, it follows that

$$
T E(\delta)=\int_{\mathbf{C}} z \chi_{\delta} d E
$$

holds for all compact subsets $\delta \subseteq \mathbf{C}$ and it follows immediately that $T=\int_{\mathbf{C}} z d E$, so that $T$ is a scalar-type spectral operator in the sense of Dunford. In the sequel, we will use the term scalar-type spectral operator without risk of confusion of terminology.

Suppose now that $P: \Sigma \rightarrow \mathscr{L}(X)$ is a closed spectral measure with $\Sigma$ a $\sigma$-algebra of subsets of some set $\Omega$, and let $\mathscr{M}$ be the range of $P$. We denote by $\mathscr{L}^{0}(P)$ the linear space of all complex valued $\Sigma$-measurable functions on $\Omega \cdot \mathscr{L}^{0}(P)$ is clearly a complex Riesz space, in fact an 
$f$-algebra with respect to the pointwise ordering, and by [6], Proposition 1.8 , it follows that the order ideal of $P$-null functions in $\mathscr{L}^{0}(P)$ is an algebraic ideal as well as an $f$-subalgebra. We denote by $L^{0}(P)$ the corresponding quotient space.

Proposition 6.1. If $P: \Sigma \rightarrow \mathscr{L}(X)$ is a closed spectral measure and if $\mathscr{M}$ is the range of $P$, then the map $P: f \mapsto \int_{\Omega} f d P, f \in \mathscr{L}^{0}(P)$ induces an f-algebra isomorphism of $L^{0}(P)$ onto $\langle\mathscr{M}\rangle_{\infty}$.

Proof. We continue to denote the induced mapping by $P$. We show first that $P$ maps $L^{0}$ onto $\langle\mathscr{M}\rangle_{\infty}$. If $T \in\langle\mathscr{M}\rangle_{\infty}$, it suffices to show that there exists $f \in \mathscr{L}^{0}(P)$ such that $T=\int_{\Omega} f d P$. From above, there exists an equicontinuous spectral measure $E$ defined on the Borel subsets $\mathscr{B}$ of the complex plane such that $T=\int_{\mathbf{C}} z d E$. Let $\left\{B_{n}\right\}$ be any sequence of compact subsets of $\mathbf{C}$ for which $B_{n} \uparrow_{n} \mathbf{C}$. For each $n=1,2, \ldots$ there exists $G_{n} \in \Sigma$ and $f_{n} \in L^{1}(P)$ such that $P\left(G_{n}\right)=E\left(B_{n}\right)$ and

$$
T E\left(B_{n}\right)=\int_{\Omega} f_{n} d P=P\left(G_{n}\right) \int_{\Omega} f_{n} d P=\int \chi_{G_{n}} f_{n} d P
$$

for $n=1,2, \ldots$. Without loss of generality, it may be assumed that $G_{n} \uparrow_{n} \Omega$ and that $f_{n}=0$ on $\Omega \backslash G_{n}, n=1,2, \ldots$ It follows that

$$
\int_{\Omega} \chi_{G_{n}} f_{n+1} d P=\int_{\Omega} f_{n} d P, \quad n=1,2, \ldots
$$

so that we may further assume that $f_{n+1}=f_{n}$ holds on $G_{n}$, for $n=1,2, \ldots$. We now define $f$ by setting $f(\omega)=f_{n}(\omega)$ if $\omega \in G_{n}$. It is clear that $f$ is $\Sigma$-measurable and from

$$
\int_{\Omega} f \chi_{G_{n}} d P=T P\left(G_{n}\right)=T E\left(B_{n}\right), \quad n=1,2, \ldots,
$$

it follows readily that $T=\int_{\Omega} f d P$. To see that $P$ is an algebra isomorphism of $L^{0}(P)$ onto $\langle\mathscr{M}\rangle_{\infty}$, suppose that $f, g \in \mathscr{L}^{0}(P)$ and let $\left\{G_{n}\right\}$ be any sequence in $\Sigma$ such that $G_{n} \uparrow_{n} \Omega$ and such that $f \chi_{G_{n}}, g \chi_{G_{n}} \in \mathscr{L}^{1}(P)$, $n=1,2, \ldots$. Since the restriction of $P$ to $L^{1}(P)$ is an algebraic isomorphism of $L^{1}(P)$ onto $\langle\mathscr{M}\rangle^{\#}$, it follows that

$$
P\left(f g \chi_{G_{n}}\right)=P\left(f \chi_{G_{n}}\right)\left(P g \chi_{G_{n}}\right)
$$

and

$$
P\left((f+g) \chi_{G_{n}}\right)=P\left(f \chi_{G_{n}}\right)+P\left(g \chi_{G_{n}}\right)
$$


for $n=1,2, \ldots$ It follows readily from the remark following Definition 2.7 that $P(f g)=P(f) \cdot P(g), P(f+g)=P(f)+P(g)$ hold in $\langle\mathscr{M}\rangle_{\infty}$. Finally, since the restriction of $P$ to $L^{1}(P)$ is a Riesz isomorphism of $L^{1}(P)$ onto $\langle\mathscr{M}\rangle^{\#}$, it follows that $P$ is a Riesz isomorphism of $L^{0}(P)$ onto $\langle\mathscr{M}\rangle_{\infty}$ and by this the proposition is completely proved.

In view of Proposition 6.1 preceding, Theorem 6.2 and Corollary 6.3 following are now no more than a reformulation of Theorem 5.7 and Corollary 5.8 above. If $P: \Sigma \rightarrow \mathscr{L}(X)$ is a spectral measure, we denote by $\mathscr{L}^{\infty}(P)$ the linear space of all bounded complex $\Sigma$-measurable functions on the underlying set $\Omega$.

TheOREM 6.2. Let $P: \Sigma \rightarrow \mathscr{L}(X)$ be a closed spectral measure and let $T$ be a densely defined operator in $\mathscr{L}(X)$ with domain $\mathscr{D}(X)$ invariant under $\int_{\Omega} f d P$ for each $f \in \mathscr{L}^{\infty}(P)$. The following statements are equivalent.

(i) $T$ leaves invariant all closed subspaces of $X$ which are invariant under the range of $P$.

(ii) $T$ is closable and there exists $f \in \mathscr{L}^{0}(P)$ such that the closure of $T$ is given by the spectral integral $\int_{\Omega} f d P$.

Corollary 6.3. Let $P: \Sigma \rightarrow \mathscr{L}(X)$ be a closed spectral measure and let $T$ be a densely defined closed linear operator in $X$ with domain $\mathscr{D}(T)$ invariant under the range of $P$. The following statements are equivalent.

(i) $T$ leaves invariant all closed subspaces of $X$ which are invariant under the range of $P$.

(ii) There exists $f \in \mathscr{L}^{0}(P)$ such that $T=\int_{\Omega} f d P$.

We remark that Corollary 6.3 above was proved by Masani and Rosenberg [13] for the case that $X$ is a Hilbert space; by Bade [2] for the case that $X$ is Banach and $T$ continuous and by Sourour [22] for the case that $X$ is Banach and $T$ is densely defined and closed. The methods of these papers do not extend to the locally convex setting. For the case that $X$ is locally convex and $T$ is continuous, then Corollary 6.3 was proved, explicitly, in [6] (Proposition 1.5 and Theorem 3.1) and, implicitly, in [4], Corollary 5.6. The methods of the present paper follow those of [4], and this approach yields the stronger result, Theorem 6.2 above, which appears to be new, even for the case that $X$ is Banach. In the Banach space setting, a special case of Theorem 6.2 may be found in [19], Theorem 7.

We turn now to questions related to the spectrum of scalar-type spectral operators. If $X$ is a Banach space and $T$ a scalar-type spectral operator on $X$, then it is well known ([7], Chapters XV, XVIII) that $T$ has 
a uniquely determined resolution of the identity which commutes with each continuous linear operator commuting with $T$ and whose support is precisely the spectrum of $T$. In the locally convex setting, these questions have been considered for continuous operators in [12] and [17], and for unbounded operators with non-empty resolvent set in [20]. We show now that these results permit exact extensions to locally convex setting. As in previous sections, our approach is via order structure and this permits a treatment which applies simultaneously to bounded and unbounded cases. While we follow as closely as possible the arguments of [7], Chapter XV, the main difference in the present approach is that we exploit the algebraic structure of the algebras $\langle\mathscr{M}\rangle_{\infty}$, where $\mathscr{M}$ is a Bade complete Boolean algebra of projections in $X$.

Let $T$ be a linear operator in $X$ with domain $\mathscr{D}(T)$. The complex number $z_{0}$ is said to belong to the resolvent set $\rho(T)$ of $T$ if there exists an open neighbourhood $U$ of $z_{0}$ such that, for all $z \in U$, the linear map $z I-T$ is injective, has dense range and $(z I-T)^{-1}$ extends to a continuous operator $R(z ; T): X \rightarrow X$, such that $R(z ; T)(z I-T)$ is the identity on $\mathscr{D}(T),(z I-T) R(z ; T)$ is the identity map of the range of $z I-T$ and such that the map $z \mapsto R(z ; T)$ is analytic on $U$. The map $R(\cdot, T)$ is called the resolvent of $T$ on $\rho(T)$. The spectrum $\sigma(T)$ of $T$ is then defined to be the complement of $\rho(T)$ in $\mathbf{C}$.

LEMMA 6.4. Let $\mathscr{M}$ be a Bade complete Boolean algebra of projections in $X$ and let $T \in\langle\mathscr{M}\rangle_{\infty}$. Let $E: \mathscr{B} \rightarrow \mathscr{L}(X)$ be the equicontinuous spectral measure on the Borel subsets $\mathscr{B}$ of the complex plane $\mathbf{C}$ generated by the Freudenthal system of $T$ in $\mathscr{M}$. If $z \in \mathbf{C}$, if $\delta \in \mathscr{B}$ and if $d(z, \delta)$ is the distance of $z$ to $\delta$, then

$$
|z E(\delta)-T E(\delta)| E(\delta) \geq d(z, \delta) E(\delta)
$$

holds in $\langle\mathscr{M}\rangle_{\infty}$

The preceding lemma may be proved by a direct application of Proposition 6.1 above. An intrinsic proof may be based alternatively on the properties of the Freudenthal spectral system as in [11] $\S 38,56$. We omit the details.

If $\mathscr{M}, T$ and $E$ are as in the statement of the preceding lemma and if $\delta$ is a Borel subset of $\mathbf{C}$, we denote by $\bar{\delta}$ the closure of $\delta$ and by $T_{\delta}, \mathscr{M}_{\delta}$ the restrictions of $T, \mathscr{M}$ to $E(\delta)(X)$ respectively. Note that $\mathscr{M}_{\delta}$ is a Bade complete Boolean algebra of projections in $E(\delta)(X)$ and that $T_{\delta} \in\langle\mathscr{M}\rangle_{\infty}$. 
Proposition 6.5. Let $\mathscr{M}$ be a Bade complete Boolean algebra of projections in $X$, let $T \in\langle\mathscr{M}\rangle_{\infty}$ and let $E: \mathscr{B} \rightarrow \mathscr{L}(X)$ be the equicontinuous spectral measure on the Borel subsets of the complex plane $\mathbf{C}$ generated by the Freudenthal system of $T$ in $\mathscr{M}$. If $\delta$ is a Borel subset of $\mathbf{C}$, then

(i) $\sigma\left(T_{\delta}\right) \subseteq \bar{\delta}$

(ii) $\left|R\left(z ; T_{\delta}\right)\right| \leq(d(z ; \delta))^{-1} I_{\delta}$ holds in $\langle\mathscr{M}\rangle_{\delta}$, whenever $z \in \mathbf{C} \backslash \bar{\delta}$.

Proof. It is clear that we may assume that $\delta$ is not dense in C. Suppose then that $\varnothing \neq U \subseteq \mathbf{C}$ is open with distance $\varepsilon>0$ to $\delta$. From Lemma 6.2, it follows that

$$
\left|z I_{\delta}-T_{\delta}\right| \geq \varepsilon I_{\delta}, \quad z \in U
$$

holds in $\left\langle\mathscr{M}_{\delta}\right\rangle_{\infty}$ and consequently, it follows from [24], Theorem 146.3 that $z I_{\delta}-T_{\delta}$ is invertible in $\left\langle\mathscr{M}_{\delta}\right\rangle_{\infty}$ with inverse $R\left(z ; T_{\delta}\right) \in\left\langle\mathscr{M}_{\delta}\right\rangle$ satisfying

$$
\left|R\left(z ; T_{\delta}\right)\right| \leq \varepsilon^{-1} I_{\delta}, \quad z \in U
$$

Since

$$
R\left(w ; T_{\delta}\right)=\sum_{n=0}^{\infty}(-1)^{n}(w-z)^{n}\left(R\left(z ; T_{\delta}\right)\right)^{n+1}
$$

holds $I_{\delta}$-uniformly in $\left\langle\mathscr{M}_{\delta}\right\rangle$ for $w, z \in U,|w-z|<\varepsilon / 2$, it follows that the map $z \rightarrow R\left(z ; T_{\delta}\right)$ is analytic in $U$. Further, since

$$
R\left(z ; T_{\delta}\right)\left(z I_{\delta}-T_{\delta}\right)=\left(z I_{\delta}-T_{\delta}\right) R\left(z ; T_{\delta}\right)=I_{\delta}
$$

holds in $\left\langle\mathscr{M}_{\delta}\right\rangle_{\infty}$, it follows that

$$
R\left(z ; T_{\delta}\right)\left(z I_{\delta}-T_{\delta}\right) x=x, \quad z \in U
$$

holds for all $x \in \mathscr{D}\left(T_{\delta}\right)$ so that $\left(z I_{\delta}-T_{\delta}\right)$ is injective for all $z \in U$; further, it follows that

$$
\left(z I_{\delta}-T_{\delta}\right) R\left(z ; T_{\delta}\right) x=x, \quad z \in U
$$

holds for all $x \in E(\delta)(X)$ for which $R\left(z ; T_{\delta}\right) x \in \mathscr{D}\left(T_{\delta}\right)$, so that $z I_{\delta}-R_{\delta}$ has dense range for all $z \in U$. It follows that $U \subseteq \rho\left(T_{\delta}\right)$ and by this the proposition is proved.

If $T, E, \mathscr{M}$ are as above, the essential step required to characterize the range in $X$ of each projection in the range of $E$ is given by the following lemma. See, for example [7] Lemma XVIII 2.3. As the proof may be based on Proposition 6.5 above and arguments similar to those of [7], XV 3.1, 3.2, 3.4, the details will be omitted. 
LEMMA 6.6. Let $\mathscr{M}$ be a Bade complete Boolean algebra of projections in $X$, let $T \in\langle\mathscr{M}\rangle_{\infty}$ and let $E: \mathscr{B} \rightarrow \mathscr{L}(X)$ be the equicontinuous spectral measure on the Borel subsets of the complex plane generated by the Freudenthal system of $T$ in $\mathscr{M}$. If $x \in \mathscr{D}(T)$, if $\delta \subseteq \mathbf{C}$ is compact and if $f$ : $\mathbf{C} \backslash \delta \rightarrow \mathscr{D}(T)$ is an analytic map for which $(z I-T)(f(z))=x$ for all $z \in \mathbf{C} \backslash \delta$, then $E(\sigma) x=x$.

The preceding lemma, combined with an inspection of the proof of [7] XV 3.7 now yields the following result. We recall first that if $A \in \mathscr{L}(X)$ and $T: \mathscr{D}(T) \rightarrow \mathscr{L}(X)$ is a linear map, then $A$ is said to commute with $T$ if and only if $A(\mathscr{D}(T)) \subseteq \mathscr{D}(T)$ and $A T x=T A x$ for all $x \in \mathscr{D}(T)$.

Proposition 6.7. Let $\mathscr{M}$ be a Bade complete Boolean algebra of projections in $X$, let $T \in\langle\mathscr{M}\rangle_{\infty}$ and let $E: \mathscr{B} \rightarrow \mathscr{L}(X)$ be the equicontinuous spectral measure on the Borel subsets of the complex plane generated by the Freudenthal system of $T$ in $\mathscr{M}$. If $A \in \mathscr{L}(X)$ commutes with $T$, then $A$ commutes with $E(\delta)$, for each Borel subset $\delta$ of the complex plane.

If $T$ is a scalar-type spectral operator in $X$, a resolution of the identity for $T$ is any equicontinuous spectral measure $F: \mathscr{B} \rightarrow \mathscr{L}(X)$ defined on the Borel subsets $\mathscr{B}$ of the complex plane such that $\int_{\mathbf{C}} z d F$ is a representation of $T$ as a spectral integral. We remark that if $F$ is a resolution of the identity for $T$ and if $\mathscr{M}$ is the closure of the range of $F$ in $\mathscr{L}(X)$, then it is a consequence of the uniqueness of the Freudenthal system ([11], Theorem 40.8$)$ that $F$ coincides with the spectral measure on $\mathscr{B}$ generated by the Freudenthal system of $T$ in $\mathscr{M}$.

Proposition 6.8. If $T$ is a scalar-type spectral operator in $X$ then $T$ has a unique resolution of the identity.

Proof. Suppose $E, F$ are resolutions of the identity for $T$. By the remark immediately preceding the proposition, it may be assumed that $E$, $F$ are generated by the Freudenthal systems of $T$ in $\langle\mathscr{M}\rangle_{\infty},\langle\mathscr{N}\rangle_{\infty}$ where $\mathscr{M}, \mathscr{N}$ denote respectively the closures of the range of $E, F$ in $\mathscr{L}(X)$. If $\delta \subseteq \mathbf{C}$ is compact then it follows from Proposition 6.5 that the restriction of $z I-T$ to $F(\delta)(X)$ is invertible for $z \in \mathbf{C} \backslash \delta$, with inverse $R\left(z ; T_{\delta}\right)$, such that $R\left(\cdot, T_{\delta}\right)$ is analytic in $\mathrm{C} \backslash \delta$. Here $T_{\delta}$ denotes the restriction of $T$ to $F(\delta)(X)$. Since

$$
(z I-T) F(\delta) R\left(z ; T_{\delta}\right) F(\delta) x=F(\delta) x
$$


holds for all $x \in X$ and $z \in \mathbf{C} \backslash \delta$, it follows from Lemma 6.6 that $E(\delta) F(\delta)=F(\delta)$. It follows similarly that $F(\delta) E(\delta)=E(\delta)$. From Proposition 6.7 , it follows that $E(\delta)=F(\delta)$ for all compact $\delta \subseteq \mathbf{C}$ and this clearly suffices to prove the Proposition.

LEMMA 6.9. If $T$ is a scalar-type spectral operator in $X$ with resolution of the identity $E$ and if $\varnothing \neq \delta \subseteq \mathbf{C}$ is compact, then the restriction of $T$ to $E(\delta)(X)$ has non-void spectrum, provided $E(\delta) \neq\{0\}$.

Proof. Denote by $T_{\delta}$ the restriction of $T$ to the range of $E(\delta)$. If $\sigma\left(T_{\delta}\right)=\varnothing$, it follows that the map $z \rightarrow R\left(z ; T_{\delta}\right)$ is entire. From Proposition 6.5(ii), it follows that

$$
\left|R\left(z ; T_{\delta}\right) x\right| \leq\left|R\left(z, T_{\delta}\right)\right| x \leq(d(z, \delta))^{-1} x
$$

holds in the Riesz space $\mathscr{M}[x]$ whenever $z \notin \delta$. Consequently $R\left(z ; T_{\delta}\right) x$ $\rightarrow 0$ as $z \rightarrow \infty$ for each $x \in X$, since $\delta$ is compact. The result now follows from Liouville's theorem.

If $T$ is a scalar-type spectral operator in $X$, with resolution of the identity $E$, we denote by $\operatorname{supp} E$ the complement in $\mathbf{C}$ of the largest open set $V$ for which $E(V)=0$.

Proposition 6.10. If $T$ is a scalar-type spectral operator in $X$ with resolution of the identity $E$, then $\sigma(T)=\operatorname{supp} E$.

Proof. Since $E(\operatorname{supp} E)=I$, it follows from Proposition 6.5(i) that $\sigma(T) \subseteq \operatorname{supp} E$. To show that $\operatorname{supp} E \subseteq \sigma(T)$, observe first that if $\sigma(T)=$ $\mathrm{C}$, there is nothing to prove. It suffices then to show that if $\rho(T) \neq \varnothing$ and if $\delta \subseteq \rho(T)$ is compact, then $E(\delta)=0$. If $\delta \subseteq \rho(T)$ is compact, it is simply checked that the restriction of the resolvent map $R(z ; T)$ to the range of $E(\delta)$ is the resolvent of the restriction $T_{\delta}$ of $T$ to the range of $E(\delta)$ so that $\sigma\left(T_{\delta}\right) \subseteq \sigma(T)$. By Proposition 6.5(i) $\sigma(T) \subseteq \delta$ so that $\sigma\left(T_{\delta}\right)=\varnothing$. This however contradicts Lemma 6.9 and by this, the proposition is proved.

We remark that if $T$ is a scalar-type spectral operator in $X$ with resolution of the identity $E$, then it follows immediately from the preceding result that

$$
\sigma(T)=\bigcap\{\bar{\delta}: \delta \text { Borel, } E(\delta)=I\} .
$$

If now $f$ is a complex-valued Borel function on $\mathbf{C}$, we denote by $f(T)$ the spectral integral $\int_{\mathbf{C}} f d E$. Just as in [7], XVII 2.17 it follows that the resolution of the identity of $f(T)$ is the spectral measure $E\left(f^{-1}(\cdot)\right)$. It 
follows that

$$
\sigma(f(T))=\bigcap\{\overline{f(\delta)}: \delta \text { Borel, } E(\delta)=I\} .
$$

In particular, it follows that $\sigma(f(T)) \subseteq \overline{f(\sigma(T))}$. Observe now that if $\delta \subseteq \mathbf{C}$ is Borel and $E(\delta)=I$, then $\delta(T) \cap \delta=\sigma(T)$. Hence, if $\delta \subseteq \mathbf{C}$ is Borel and $E(\delta)=I$, and if $f$ is continuous on $\sigma(T)$, then

$$
f(\sigma(T))=f(\overline{\sigma(T) \cap \delta)} \subseteq \overline{f(\sigma(T) \cap \delta)} \subseteq f(\sigma)
$$

so that $f(\sigma(T)) \subseteq \sigma(T)$. These remarks constitute the proof of the following spectral mapping theorem (cf. [17] Theorem 2).

Proposition 6.11. If $T$ is a scalar-type spectral operator in $X$ and if $f$ : $\mathrm{C} \rightarrow \mathrm{C}$ is a complex Borel function which is continuous on $\sigma(T)$ then $\sigma(f(T))=\overline{f(\sigma(T))}$.

The final result of this section may be proved by an appropriate modification of the proof of [23], Theorem XI 7.1 and accordingly we omit the details. Note, however, that the reasoning of the first part of the proof of Proposition 6.1 does not apply.

Proposition 6.12. If $S, T$ are scalar-type spectral operators in $X$ with resolutions of the identity $F, E$ respectively then there exists a complex Borel function $f: \mathbf{C} \rightarrow \mathbf{C}$ such that $S=f(T)$ if and only if the range of $F$ is contained in the range of $E$.

7. Concluding remarks. The results of the preceding sections, together with the main reflexivity theorem of [5], provide the key tools necessary to extend to the locally convex setting certain theorems concerning (not necessarily commutative) reflexive algebras of operators due to Sourour [21] and Rosenthal and Sourour [19].

We begin with the following complement to, and consequence of, Theorem 3.8 of [5]. If $\mathscr{A} \subseteq \mathscr{L}(X)$, the double commutant of $\mathscr{A}$ is the set of all continuous linear operators on $X$ which commute with each continuous operator commuting with $\mathscr{A}$. If $\mathscr{A}$ is a unital subalgebra of $\mathscr{L}(X)$ then $\mathscr{A}$ is called reflexive if $\mathscr{A}$ contains each continuous linear operator which leaves invariant each $\mathscr{A}$-invariant subspace.

Proposition 7.1. Let $\mathscr{M}$ be a Bade complete Boolean algebra of projections in $X$ and let $\mathscr{A}$ be a closed unital subalgebra of $\langle\mathscr{M}\rangle$. If each $\mathscr{A}$-invariant subspace is range of a continuous projection which commutes with $\mathscr{A}$ then

(i) $\mathscr{A}$ is equal to its double commutant.

(ii) There exists a Bade complete Boolean subalgebra $\mathscr{N} \subseteq \mathscr{M}$ such that $\mathscr{A}=\langle\mathscr{N}\rangle$. 
Proof. (i) It follows immediately that each element of the double commutant of $\mathscr{A}$ leaves invariant each $\mathscr{A}$-invariant subspace and hence belongs to $\mathscr{A}$, since $\mathscr{A}$ is reflexive, by [5], Theorem 3.8 .

(ii) From Proposition 6.7 it follows that the spectral resolution of each element of $\mathscr{A}$ lies in the double commutant of $\mathscr{A}$ hence in $\mathscr{A}$, by (i). It follows that the Boolean subalgebra $\mathscr{N}_{0}$ of $\mathscr{M}$ generated by the set of all projections in the ranges of the spectral resolutions of all elements of $\mathscr{A}$ is again contained in $\mathscr{A}$. If $\mathscr{N}$ is the closure of $\mathscr{N}_{0}$, then $\mathscr{N}$ is Bade complete by [4], Proposition 4.5 and it is clear that $\mathscr{A}=\langle\mathscr{N}\rangle$.

We remark that if $X$ is Banach, then the preceding result was proved by Sourour [21], Theorem 8 , under the additional assumption that the Boolean algebra $\mathscr{M}$ is of uniform multiplicity one (see below).

The papers [18], [19] are concerned with the extension to the Banach space setting of reflexivity results for strongly closed algebras of operators on, in general, non separable Hilbert spaces, which contains a maximal abelian von Neumann algebra. If $X$ is a Banach space, a Bade complete Boolean algebra of projections in $X$ is said to be of uniform multiplicity one if the restriction of $\mathscr{M}$ to $E(X)$ has a cyclic vector whenever $E \in \mathscr{M}$ is countably decomposable (the projection $E \in \mathscr{M}$ is said to be countably decomposable if every family of disjoint projections in $\mathscr{M}$ majorized by $E$ is at most countable). We observe the following simple characterization of Boolean algebras of uniform multiplicity one.

LeMma 7.2. Let $X$ be a Banach space and let $\mathscr{M}$ be a Bade complete Boolean algebra of projections in $X$. The following statements are equivalent:

(i) $\mathscr{M}$ is of uniform multiplicity one.

(ii) For each $x \in X$, there exists a projection $E \in \mathscr{M}$ such that $E(X)=$ $\mathscr{M}[x]$.

Proof. (i) $\Rightarrow$ (ii). Suppose that $\mathscr{M}$ is of uniform multiplicity one, let $x \in X$, and let $E_{x}$ be the carrier of $x$ in $\mathscr{M}$. By [7], Lemma XVIII 3.5, $E_{x}$ is countably decomposable so that, by (i), there exists $y \in X$ with $E_{x} y=y$ such that $\left(E_{x} \mathscr{M}\right)[y]=E_{x}(X)$. Without loss of generality, we may assume that $E_{x}=I$ and that $y$ is cyclic for $\mathscr{M}$. From Propositions 2.1, 2.4 (ii) of [5] it follows that $\mathscr{M}[x]$ is a band in the Riesz space $\mathscr{M}[y]$ and that the corresponding band projection is given by an element of $\mathscr{M}$. It now follows from the fact that $E_{x}=I$, that $\mathscr{M}[x]=\mathscr{M}[y]$ and so the implication (i) $\Rightarrow$ (ii) follows. 
(ii) $\Rightarrow$ (i). If $E \in \mathscr{M}$ is countably decomposable, then by [7], Lemma XVIII 3.5, it follows that $E$ is the carrier projection $E_{x}$ of some element $x \in X$. By (i), there exists a projection $F \in \mathscr{M}$ such that $F(X)=\mathscr{M}[x]$. Necessarily $F=E_{x}=E$ and by this, the implication is proved.

If now $X$ is a locally convex quasicomplete space, a Bade complete Boolean algebra of projections in $X$ will be called quasi-cyclic if condition (ii) of the preceding Lemma 7.2 is valid. It follows from Propositions 2.4, 2.7 of [5] that if $\mathscr{M}$ is a Bade complete quasi-cyclic Boolean algebra of projections in $X$, then $X$ admits the structure of a locally solid topologically complete, Dedekind complete Riesz space with Lebesgue topology such that the Boolean algebra of band projections coincides with the Boolean algebra $\mathscr{M}$ and such that the closed $\mathscr{M}$-invariant subspaces of $X$ are precisely the bands in $X$. If now $\mathscr{A}$ is a strongly closed subalgebra of $\mathscr{L}(X)$ which contains a Bade complete quasi-cyclic Boolean algebra of projections then each $\mathscr{A}$-invariant subspace, being $\mathscr{M}$-invariant, is the range of a projection in $\mathscr{M}$ and it follows that each $\mathscr{A}$-invariant subspace which has an $\mathscr{A}$-invariant complement is the range of projection in $\mathscr{M}$ which commutes with $\mathscr{A}$. We may now state the following extension of [19] Theorem 5.

Proposition 7.3. Let $\mathscr{A}$ be a strongly closed subalgebra of $\mathscr{L}(X)$ which contains a Bade complete quasi-cyclic Boolean algebra of projections. If every invariant subspace of $\mathscr{A}$ has an invariant complement then

(i) $\mathscr{A}$ is equal to its double commutant.

(ii) $\mathscr{A}$ is reflexive.

The proof of the preceding proposition now follows exactly as in [19], with Theorem 7 of [19] replaced by Theorem 5.7 of the present paper. We omit the details.

In a similar vein, we leave to the interested reader the task of checking that the tools provided by the present paper are sufficient to extend verbatim to the locally convex setting the proofs in [18] of the following results for Banach spaces due to Rosenthal and Sourour. See [18], Theorem 8.12.

Proposition 7.4. If $\mathscr{A}$ is a closed subalgebra of $\mathscr{L}(X)$ which contains a Bade complete quasi-cyclic Boolean algebra of projections and if the only $\mathscr{A}$-invariant subspaces are $\{0\}$ and $X$, then $\mathscr{A}=\mathscr{L}(X)$. 
Proposition 7.5. If $\mathscr{A}$ is a closed subalgebra of $\mathscr{L}(X)$ which contains a Bade complete quasi-cyclic Boolean algebra of projections, and if the $\mathscr{A}$-invariant subspaces of $X$ are totally ordered, then $\mathscr{A}$ is reflexive.

\section{REFERENCES}

[1] C. D. Aliprantis and O. Burkinshaw, Locally Solid Riesz Spaces, New York-San Francisco-London: Academic Press 1978.

[2] W. G. Bade, On Boolean algebras of projections and algebras of operators, Trans. Amer. Math. Soc., 80 (1955), 343-359.

[3] P. G. Dodds, Orthomorphisms of a commutative $W^{*}$-algebra, J. Austral. Math. Soc. (Series A), 35 (1983), 143-168.

[4] P. G. Dodds and B. de Pagter, Orthomorphisms and Boolean algebras of projections, Math. Z., 187 (1984), 361-381.

[5] P. G. Dodds, B. de Pagter and W. Ricker, Reflexivity and order properties of scalar-type spectral operators in locally convex spaces, Trans. Amer. Math. Soc., 293 (1986), 355-380.

[6] P. G. Dodds and W. Ricker, Spectral measures and the Bade reflexivity theorem, J. Functional Anal., 61 (1985), 136-163.

[7] N. Dunford and J. Schwartz, Linear operators Part III. Spectral Operators, New York: Wiley-Interscience 1971.

[8] I. Kluvànek, The extension and closure of vector measures, in Vector and Operator-valued Measures and Applications, pp. 175-189. New York: Academic Press 1973.

[9] W. A. J. Luxemburg, Some aspects of the theory of Riesz spaces, University of Arkansas Lecture Notes in Mathematics 4: Fayetteville 1979.

[10] W. A. J. Luxemburg and A. R. Schep, A Radon-Nikodym theorem for positive operators and a dual, Nederl. Akad. Wetens. Proc. Ser. A, 81 (1978), 357-375.

[11] W. A. J. Luxemburg and A. C. Zaanen, Riesz Spaces I, Amsterdam-London: North Holland, 1971.

[12] F. Maeda, Spectral theory in locally convex spaces, Ph.D. Thesis, Yale University 1961.

[13] P. Masani and M. Rosenberg, When is an operator the integral of a given spectral measure?, J. Functional Anal., 21 (1976), 88-121.

[14] B. de Pagter, Disjointness preserving operators, Proc. Amer. Math. Soc., 90 (1984), 543-549.

[15] _ The space of extended orthomorphisms in a Riesz space, Pacific J. Math., 112 (1984), 193-210.

[16] W. Ricker, On Boolean algebras of projections and scalar-type spectral operators, Proc. Amer. Math. Soc., 87 (1983), 73-77.

[17] A A spectral mapping theorem for scalar-type spectral operators in locally convex spaces, Integral Equations Operator Theory, 8 (1985), 276-288.

[18] P. Rosenthal and A. R. Sourour, On operator algebras containing cyclic Boolean algebras I, Pacific J. Math., 70 (1977), 243-252.

[19] , On operator algebras containing cyclic Boolean algebra II, J. London Math. Soc., 16 (1977), 501-506.

[20] H. H. Schaefer, Spectral measures in locally convex algebras, Acta Math., 107 (1962), 125-173.

[21] A. R. Sourour, On algebras of Banach space operators and invariant subspaces, Bull. London Math. Soc., 9 (1977), 305-309. 
[22] __ Unbounded operators generated by a given spectral measure, J. Functional Analysis, 29 (1978), 16-22.

[23] B. Z. Vulikh, Introduction to the Theory of Partially Ordered Spaces, WoltersNoordhoff, Groningen, 1967.

[24] A. C. Zaanen, Riesz Spaces II, North-Holland, Amsterdam-New York-Oxford, 1983.

Received April 17, 1986. Work of the second author on this paper was supported by the Netherlands Organization for the Advancement of Pure Research (Z.W.O.).

INSTITUT FUR MATHEMATIK

JOHANNES KEPLER UNIVERSITÄT LINZ

LiNZ, AUSTRIA

School of Mathematical Sciences

The Flinders UnIVERSITY OF SOUTH Australia

BEDFORD PARK S. A. 5042

Australia

AND

DePaRTMENT OF MATHEMATICS

DELFT UNIVERSITY OF TECHNOLOGY

JulianalaAN 132, 2628 BL DeLFT

THE NETHERLANDS 


\section{PACIFIC JOURNAL OF MATHEMATICS EDITORS}

V. S. VARADARAJAN (Managing Editor) University of California Los Angeles, CA 90024 HERBERT ClEMENS University of Utah Salt Lake City, UT 84112

R. FINN

Stanford University

Stanford, CA 94305

\section{HERMANN FLASCHKA} University of Arizona Tucson, AZ 85721

RAMEsh A. GANGolli University of Washington Seattle, WA 98195

VAUghan F. R. JONES

University of California Berkeley, CA 94720

\section{ROBION KIRBY}

University of California

Berkeley, CA 94720

C. C. MOORE

University of California

Berkeley, CA 94720

HAROLD STARK

University of California, San Diego

La Jolla, CA 92093

\section{ASSOCIATE EDITORS}
R. ARENS
E. F. BECKENBACH
B. H. NEUMANN
F. WOLF
K. YOSHIDA (1906-1982)

\section{SUPPORTING INSTITUTIONS}

UNIVERSITY OF ARIZONA

UNIVERSITY OF BRITISH COLUMBIA

CALIFORNIA INSTITUTE OF TECHNOLOGY

UNIVERSITY OF CALIFORNIA

MONTANA STATE UNIVERSITY

UNIVERSITY OF NEVADA, RENO

NEW MEXICO STATE UNIVERSITY

OREGON STATE UNIVERSITY
UNIVERSITY OF OREGON

UNIVERSITY OF SOUTHERN CALIFORNIA

STANFORD UNIVERSITY

UNIVERSITY OF HAWAII

UNIVERSITY OF TOKYO

UNIVERSITY OF UTAH

WASHINGTON STATE UNIVERSITY

UNIVERSITY OF WASHINGTON 


\section{Pacific Journal of Mathematics}

Vol. 130, No. $1 \quad$ September, 1987

K. Adachi, Continuation of bounded holomorphic functions from certain subvarieties to weakly pseudoconvex domains $\ldots \ldots \ldots \ldots \ldots \ldots \ldots$

Erazm Jerzy Behr, Enveloping algebras of Lie superalgebras ........... 9

Dong M. Chung, Scale-invariant measurability in abstract Wiener spaces . . . 27

Peter Gerard Dodds and Bernardus de Pagter, Algebras of unbounded

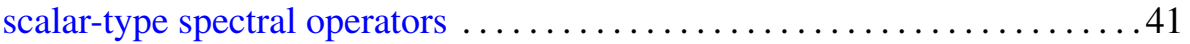

Wu-Yi Hsiang and Hsueh-Ling Huynh, Generalized rotational

hypersurfaces of constant mean curvature in the Euclidean spaces. II . . . .75

Harvey Bayard Keynes and M. Sears, Time changes for $\mathbf{R}^{n}$ flows and suspensions ..................................... 97

Frances Kirwan, Ronnie Lee and Steven Howard Weintraub, Quotients

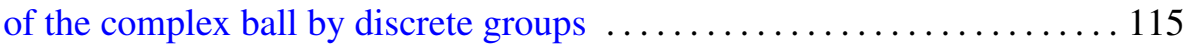

Magnhild Lien, Groups of knots in homology 3-spheres that are not

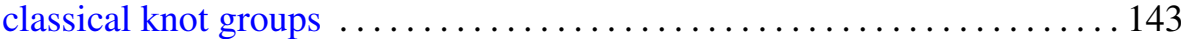

Juan Carlos Migliore, Liaison of a union of skew lines in $\mathbf{P}^{4} \ldots \ldots \ldots \ldots 153$

Jesper M. Møller, Spaces of sections of Eilenberg-Mac Lane fibrations . . . . 171 Daniel Ullman, A generalization of a theorem of Atkinson to noninvariant

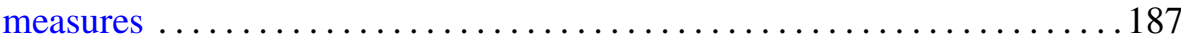

Kohhei Yamaguchi, Operations which detect $\mathscr{P}^{1}$ in odd primary connective

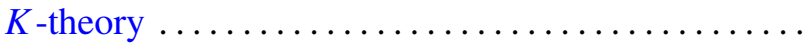

